ANALYSIS \& PDE

Volume $4 \quad$ No. $3 \quad 2011$

\author{
OANA POCOVNICU
}

TRAVELING WAVES FOR THE CUBIC SZEGÓ EQUATION ON THE REAL LINE 


\title{
TRAVELING WAVES FOR THE CUBIC SZEGŐ EQUATION ON THE REAL LINE
}

\author{
OANA POCOVNICU
}

\begin{abstract}
We consider the cubic Szegő equation $i \partial t u=\Pi\left(|u|^{2} u\right)$ in the Hardy space $L_{+}^{2}(\mathbb{R})$ on the upper half-plane, where $\Pi$ is the Szegő projector. It was first introduced by Gérard and Grellier as a toy model for totally nondispersive evolution equations. We show that the only traveling waves are of the form $C /(x-p)$, where $p \in \mathbb{C}$ with $\operatorname{Im} p<0$. Moreover, they are shown to be orbitally stable, in contrast to the situation on the unit disk where some traveling waves were shown to be unstable.
\end{abstract}

\section{Introduction}

One of the most important properties in the study of nonlinear Schrödinger equations (NLS) is dispersion. It is often exhibited in the form of the Strichartz estimates of the corresponding linear flow. In case of the cubic NLS,

$$
i \partial_{t} u+\Delta u=|u|^{2} u, \quad(t, x) \in \mathbb{R} \times M,
$$

Burq, Gérard, and Tzvetkov [Burq et al. 2005] observed that the dispersive properties are strongly influenced by the geometry of the underlying manifold $M$. Taking this idea further, Gérard and Grellier [2010b] remarked a lack of dispersion when $M$ is a sub-Riemannian manifold (for example, the Heisenberg group). In this situation, many of the classical arguments used in the study of NLS no longer hold. As a consequence, even the problem of global well-posedness of (1-1) on a sub-Riemannian manifold still remains open.

Gérard and Grellier [2010a; 2010b] introduced a model of a nondispersive Hamiltonian equation called the cubic Szegó equation. (See (1-2) below.) The study of this equation is the first step toward understanding existence and other properties of smooth solutions of NLS in the absence of dispersion. Remarkably, the Szegó equation turned out to be completely integrable in the following sense. It possesses a Lax pair structure and an infinite sequence of conservation laws. Moreover, the dynamics can be approximated by a sequence of finite-dimensional completely integrable Hamiltonian systems. To illustrate the degeneracy of this completely integrable structure, several instability phenomena were established in [Gérard and Grellier 2010a].

Gérard and Grellier studied the Szegô equation on the circle $\mathbb{S}^{1}$. More precisely, solutions were considered to belong at all time to the Hardy space $L_{+}^{2}\left(\mathbb{S}^{1}\right)$ on the unit disk $\mathbb{D}=\{|z|<1\}$. This is the space of $L^{2}$-functions on $\mathbb{S}^{1}$ with $\hat{f}(k)=0$ for all $k<0$. These functions can be extended as

MSC2000: 35B15, 37K10, 47B35.

Keywords: nonlinear Schrödinger equations, Szegő equation, integrable Hamiltonian systems, Lax pair, traveling wave, orbital stability, Hankel operators. 
holomorphic functions on the unit disk. Several properties of the Hardy space on the unit disk naturally transfer to the Hardy space $L_{+}^{2}(\mathbb{R})$ on the upper half-plane $\mathbb{C}_{+}=\{z ; \operatorname{Im} z>0\}$, defined by

$$
L_{+}^{2}(\mathbb{R})=\left\{f \text { holomorphic on } \mathbb{C}_{+} ;\|g\|_{L_{+}^{2}(\mathbb{R})}:=\sup _{y>0}\left(\int_{\mathbb{R}}|g(x+i y)|^{2} d x\right)^{1 / 2}<\infty\right\} .
$$

In view of the Paley-Wiener theorem, we identify this space of holomorphic functions on $\mathbb{C}_{+}$with the space of its boundary values:

$$
L_{+}^{2}(\mathbb{R})=\left\{f \in L^{2}(\mathbb{R}) ; \operatorname{supp} \hat{f} \subset[0, \infty)\right\} .
$$

The transfer from $L_{+}^{2}\left(\mathbb{S}^{1}\right)$ to $L_{+}^{2}(\mathbb{R})$ is made by the usual conformal transformation

$$
\omega: \mathbb{D} \rightarrow \mathbb{C}_{+}, \quad \omega(z)=i \frac{1+z}{1-z} .
$$

However, the image of a solution of the Szegô equation on $\mathbb{S}^{1}$ under the conformal transformation is no longer a solution of the Szegő equation on $\mathbb{R}$. Therefore, we study the Szegó equation on $\mathbb{R}$ directly.

Endowing $L^{2}(\mathbb{R})$ with the usual scalar product $(u, v)=\int_{\mathbb{R}} u \bar{v}$, we define the Szegő projector $\Pi$ : $L^{2}(\mathbb{R}) \rightarrow L_{+}^{2}(\mathbb{R})$ to be the projector onto the nonnegative frequencies:

$$
\Pi(f)(x)=\frac{1}{2 \pi} \int_{0}^{\infty} e^{i x \xi} \hat{f}(\xi) d \xi .
$$

For $u \in L_{+}^{2}(\mathbb{R})$, we consider the Szegó equation on the real line:

$$
i \partial_{t} u=\Pi\left(|u|^{2} u\right), \quad x \in \mathbb{R} .
$$

This is a Hamiltonian evolution associated to the Hamiltonian

$$
E(u)=\int_{\mathbb{R}}|u|^{4} d x
$$

defined on $L_{+}^{4}(\mathbb{R})$. From this structure, we obtain the formal conservation law

$$
E(u(t))=E(u(0))
$$

The invariance under translations and under modulations provides two more conservation laws,

$$
Q(u(t))=Q(u(0)) \quad \text { and } \quad M(u(t))=M(u(0)),
$$

where

$$
Q(u)=\int_{\mathbb{R}}|u|^{2} d x \quad \text { and } \quad M(u)=\int_{\mathbb{R}} \bar{u} D u d x, \text { with } D=-i \partial_{x} .
$$

Now, we define the Sobolev spaces $H_{+}^{s}(\mathbb{R})$ for $s \geq 0$ :

$$
H_{+}^{s}(\mathbb{R})=\left\{h \in L_{+}^{2}(\mathbb{R}) ;\|h\|_{H_{+}^{s}}:=\left(\frac{1}{2 \pi} \int_{0}^{\infty}\left(1+|\xi|^{2}\right)^{s}|\hat{h}(\xi)|^{2} d \xi\right)^{1 / 2}<\infty\right\} .
$$


Similarly, we define the homogeneous Sobolev norm for $h \in \dot{H}_{+}^{s}$ by

$$
\|h\|_{\dot{H}_{+}^{s}}:=\left(\frac{1}{2 \pi} \int_{0}^{\infty}|\xi|^{2 s}|\hat{h}(\xi)|^{2}\right)^{1 / 2}<\infty .
$$

Slight modifications of the proof of the corresponding result in [Gérard and Grellier 2010a] lead to this well-posedness result:

Theorem 1.1. The cubic Szegó equation (1-2) is globally well-posed in $H_{+}^{s}(\mathbb{R})$ for $s \geq \frac{1}{2}$. That is, given $u_{0} \in H_{+}^{1 / 2}$, there exists a unique global-in-time solution $u \in C\left(\mathbb{R} ; H_{+}^{1 / 2}\right)$ of $(1-2)$ with initial condition $u_{0}$. Moreover, if $u_{0} \in H_{+}^{s}$ for some $s>\frac{1}{2}$, then $u \in C\left(\mathbb{R} ; H_{+}^{s}\right)$.

In this paper, we concentrate on the study of traveling waves. The two main goals are the classification of traveling waves and their stability. As a result, we show that the situation on the real line is essentially different from that on the circle.

A solution for the cubic Szegó equation on the real line (1-2) is called a traveling wave if there exist $c, \omega \in \mathbb{R}$ such that

$$
u(t, z)=e^{-i \omega t} u_{0}(z-c t), \quad z \in \mathbb{C}_{+} \cup \mathbb{R}, t \in \mathbb{R}
$$

for some $u_{0} \in H_{+}^{1 / 2}(\mathbb{R})$. Note that a solution to (1-2) in $H_{+}^{1 / 2}(\mathbb{R})$ has a natural extension onto $\mathbb{C}_{+}$, and we have used this viewpoint in (1-3). Substituting (1-3) into (1-2), we see that $u_{0}$ satisfies on $\mathbb{R}$ the equation

$$
c D u_{0}+\omega u_{0}=\Pi\left(\left|u_{0}\right|^{2} u_{0}\right)
$$

In the following, we use the simpler notation $u$ instead of $u_{0}$ when we study time-independent problems. From (1-4), we see that traveling waves with nonzero velocity, $c \neq 0$, have good regularity. Indeed, we prove that $u \in H_{+}^{s}(\mathbb{R})$ for all $s \geq 0$ in Lemma 3.1. In particular, by Sobolev embedding theorem, we have $u \in L_{+}^{p}(\mathbb{R})$ for $2 \leq p \leq \infty$. On the other hand, (1-4) yields in Lemma 4.1 that there exist no nontrivial stationary waves, i.e. traveling waves of velocity $c=0$, in $L_{+}^{2}$.

Now, we present our main results:

Theorem 1.2. A function $u \in C\left(\mathbb{R}, H_{+}^{1 / 2}(\mathbb{R})\right)$ is a traveling wave if and only if there exist $C, p \in \mathbb{C}$ with Im $p<0$ such that

$$
u(0, z)=\frac{C}{z-p} .
$$

Theorem 1.3. Let $a>0, r>0$, and consider the cylinder

$$
C(a, r)=\left\{\frac{\alpha}{z-p} ;|\alpha|=a, \operatorname{Im} p=-r\right\} .
$$

Let $\left\{u_{0}^{n}\right\} \subset H_{+}^{1 / 2}$ with

$$
\inf _{\phi \in C(a, r)}\left\|u_{0}^{n}-\phi\right\|_{H_{+}^{1 / 2}} \rightarrow 0 \text { as } n \rightarrow+\infty
$$

and let $u^{n}$ denote the solution to (1-2) with initial data $u_{0}^{n}$. Then

$$
\sup _{t \in \mathbb{R}} \inf _{\phi \in C(a, r)}\left\|u^{n}(t, x)-\phi(x)\right\|_{H_{+}^{1 / 2}} \rightarrow 0 .
$$


Let us compare our results to those in [Gérard and Grellier 2010a]. In the case of the Szegó equation on $\mathbb{S}^{1}$, the nontrivial stationary waves $(c=0)$ are finite Blaschke products of the form

$$
\alpha \prod_{j=1}^{N} \frac{z-p_{j}}{1-p_{j} z},
$$

where $|\alpha|^{2}=\omega, N \in \mathbb{N}$, and $p_{1}, p_{2}, \ldots, p_{N} \in \mathbb{D}$, and the traveling waves with nonzero velocity are rational functions of the form

$$
\frac{C z^{l}}{z^{N}-p},
$$

where $N \in \mathbb{N}, l \in\{0,1, \ldots, N-1\}, C, p \in \mathbb{C}$, and $|p|>1$. Moreover, instability phenomena were displayed for some of the above traveling waves. For the cubic Szegó equation on $\mathbb{R}$, Theorems 1.2 and 1.3 state that there exist fewer traveling waves - corresponding to $N=1$ and $l=0$ in (1-6) - and that there is no instability phenomenon.

The proof of Theorem 1.2 involves arguments from several areas of analysis: a Kronecker-type theorem, scattering theory, existence of a Lax pair structure, a theorem by Lax on invariant subspaces of the Hardy space, and canonical factorization of Beurling-Lax inner functions. We now introduce the main notions and known results, and briefly describe the strategy of the proof.

As in [Gérard and Grellier 2010a], an important property of the Szegó equation on $\mathbb{R}$ is the existence of a Lax pair structure. Using the Szegó projector, we first define two important classes of operators on $L_{+}^{2}$ : the Hankel and Toeplitz operators. We use these operators to find a Lax pair. See Proposition 1.4.

A Hankel operator $H_{u}: L_{+}^{2} \rightarrow L_{+}^{2}$ of symbol $u \in H_{+}^{1 / 2}$ is defined by

$$
H_{u}(h)=\Pi(u \bar{h}) .
$$

$H_{u}$ is $\mathbb{C}$-antilinear and satisfies

$$
\left(H_{u}\left(h_{1}\right), h_{2}\right)=\left(H_{u}\left(h_{2}\right), h_{1}\right)
$$

In Lemma 3.5 below we prove that $H_{u}$ is a Hilbert-Schmidt operator of Hilbert-Schmidt norm

$$
\frac{1}{\sqrt{2 \pi}}\|u\|_{\dot{H}^{1 / 2}} \text {. }
$$

A Toeplitz operator $T_{b}: L_{+}^{2} \rightarrow L_{+}^{2}$ of symbol $b \in L^{\infty}(\mathbb{R})$ is defined by

$$
T_{b}(h)=\Pi(b h) .
$$

$T_{b}$ is $\mathbb{C}$-linear. Moreover, $T_{b}$ is self-adjoint if and only if $b$ is real-valued.

Proposition 1.4. Let $u \in C\left(\mathbb{R} ; H_{+}^{s}\right)$ for some $s>\frac{1}{2}$. The cubic Szegó equation (1-2) is equivalent to the evolution equation

$$
\frac{d}{d t} H_{u}=\left[B_{u}, H_{u}\right]
$$

where $B_{u}=\frac{i}{2} H_{u}^{2}-i T_{|u|^{2}}$. In other words, the pair $\left(H_{u}, B_{u}\right)$ is a Lax pair for the cubic Szegó equation on the real line. 
The proof of Proposition 1.4 follows the same lines as that of the corresponding result on $\mathbb{S}^{1}$ in [Gérard and Grellier 2010a], and is based on the identity

$$
H_{\Pi\left(|u|^{2} u\right)}=T_{|u|^{2}} H_{u}+H_{u} T_{|u|^{2}}-H_{u}^{3} .
$$

Combining (1-4) and (1-9), we deduce that if $u$ is a traveling wave with $c \neq 0$, then the identity

$$
A_{u} H_{u}+H_{u} A_{u}+\frac{\omega}{c} H_{u}+\frac{1}{c} H_{u}^{3}=0,
$$

holds, where

$$
A_{u}=D-\frac{1}{c} T_{|u|^{2}}
$$

In Section 2, we prove a Kronecker-type theorem for the Hardy space $L_{+}^{2}(\mathbb{R})$, where we classify all the symbols $u$ such that the operator $H_{u}$ has finite rank. The classical theorem for $L_{+}^{2}\left(\mathbb{S}^{1}\right)$ is due to Kronecker. For a proof, see [Gérard and Grellier 2010a].

We prove Theorem 1.2 in Section 4. We first prove that all traveling waves are rational functions. On $\mathbb{S}^{1}$, this follows easily from the Kronecker theorem and the fact that the operator $A_{u}$ has discrete spectrum. On $\mathbb{R}$, however, it turns out that $A_{u}$ has continuous spectrum. Therefore, we use scattering theory to study the spectral properties of $A_{u}$ in detail in Section 3. More precisely, we show that the generalized wave operators $\Omega^{ \pm}\left(D, A_{u}\right)$, rigorously defined by (3-1) below, exist and are complete. As a result, we obtain that

$$
\mathscr{H}_{\mathrm{ac}}\left(A_{u}\right) \subset \operatorname{Ker} H_{u},
$$

where $\mathscr{H}_{\mathrm{ac}}\left(A_{u}\right)$ is the absolutely continuous subspace of $A_{u}$. The subspace Ker $H_{u}$ plays an important role in our analysis. More precisely, it turns out to be invariant under multiplication by $e^{i \alpha x}$, for all $\alpha \geq 0$. Therefore, applying a theorem by Lax (Proposition 4.4) on invariant subspaces, it results that

$$
\operatorname{Ker} H_{u}=\phi L_{+}^{2},
$$

where $\phi$ is an inner function in the sense of Beurling and Lax, i.e., a bounded holomorphic function on $\mathbb{C}_{+}$such that $|\phi(x)|=1$ for all $x \in \mathbb{R}$. Using the Lax pair structure and the identity (1-10), we show that $\phi$ satisfies the simple equation

$$
c D \phi=|u|^{2} \phi
$$

However, as an inner function, $\phi$ satisfies a canonical factorization (4-3). From this, it follows that $\phi$ belongs to a special class of inner functions, the finite Blaschke products, i.e.,

$$
\phi(z)=\prod_{j=1}^{N} \frac{z-\lambda_{j}}{z-\bar{\lambda}_{j}}
$$

where $N \in \mathbb{N}$ and $\operatorname{Im} \lambda_{j}>0$ for all $j=1,2, \ldots, N$. The Kronecker-type theorem then yields that the traveling wave $u$ is a rational function. In the case of $\mathbb{S}^{1}$, the natural shift, multiplication by $e^{i x}$, was used in concluding traveling waves are of the form (1-6). In our case, we use the "infinitesimal" shift, multiplication by $x$, to show that traveling waves are of the form (1-5). 
Finally, we prove Theorem 1.3 in Section 5. The orbital stability of traveling waves is a consequence of the fact that traveling waves are ground states for the following inequality, an analogue of the sharp Gagliardo-Nirenberg inequality given in [Weinstein 1982].

Proposition 1.5. For all $u \in H_{+}^{1 / 2}(\mathbb{R})$, the following Gagliardo-Nirenberg inequality holds:

$$
\|u\|_{L^{4}} \leq \frac{1}{\sqrt[4]{\pi}}\|u\|_{L^{2}}^{1 / 2}\|u\|_{\dot{H}_{+}^{1 / 2}}^{1 / 2}
$$

or, equivalently,

$$
E \leq \frac{1}{\pi} M Q
$$

Equality holds if and only if $u=\frac{C}{x-p}$, where $C, p \in \mathbb{C}$ with $\operatorname{Im} p<0$.

Remark 1.6. Using Proposition 1.5, one can verify that the functions $u=C /(x-p)$, with $\operatorname{Im} p<0$, are indeed initial data for traveling waves. More precisely, since they are minimizers of the functional

$$
v \in H_{+}^{1 / 2} \mapsto M(v) Q(v)-\pi E(v),
$$

the differential of this functional at $u$ is zero. Thus,

$$
\frac{1}{2} Q(u) D u+\frac{1}{2} M(u) u-\pi \Pi\left(|u|^{2} u\right)=0 .
$$

Consequently, $u$ is a solution of (1-4) with

$$
c=\frac{Q(u)}{2 \pi}=\frac{|C|^{2}}{-2 \operatorname{Im} p}, \quad \omega=\frac{M(u)}{2 \pi}=\frac{|C|^{2}}{4(\operatorname{Im} p)^{2}},
$$

and hence it is an initial datum for a traveling wave.

In the case of $\mathbb{S}^{1}$, the Gagliardo-Nirenberg inequality suffices to conclude the stability of the traveling waves with $N=1$. However, in the case of $\mathbb{R}$, we need to use in addition a concentration-compactness argument. This concentration-compactness argument, which first appeared in [Cazenave and Lions 1982], was refined and turned into profile decomposition theorems by Gérard [1998] and later by Hmidi and Keraani [2006]. We use it in the form of Proposition 5.1, a profile decomposition theorem for bounded sequences in $H_{+}^{1 / 2}$.

We conclude this introduction by presenting two open problems. Here, we use the term soliton instead of traveling wave, so that we put into light several connections with existing works. The first problem is the soliton resolution, which consists in writing any solution as a superposition of solitons and radiation. For the KdV equation, this property was rigorously stated in [Eckhaus and Schuur 1983] for initial data to which the Inverse Scattering Transform applies. Therefore, for the Szegó equation, one needs to solve inverse spectral problems for the Hankel operators and also find explicit action angle coordinates.

The second open problem is the interaction of solitons with external potentials. Consider the Szegó equation with a linear potential, where initial data are taken to be of the form (1-5). As in [Holmer and Zworski 2008] and [Perelman 2009], it would be interesting to investigate if solutions of the perturbed Szegő equation can be approximated by traveling wave solutions to the original Szegő equation (1-2). 


\section{A Kronecker-type theorem}

A theorem by Kronecker asserts in the setting of $\mathbb{S}^{1}$ that the set of symbols $u$ such that $H_{u}$ is of rank $N$ is precisely a $2 N$-dimensional complex submanifold of $L_{+}^{2}\left(\mathbb{S}^{1}\right)$ containing only rational functions. In this section, we prove the analogue of this. For a different proof of a similar result on some Hankel operators on $L_{+}^{2}(\mathbb{R})$ defined in a slightly different way, see [Peller 2003, Lemma 8.12, p. 54].

Definition. Let $N \in \mathbb{N}^{*}$. We denote by $M(N)$ the set of rational functions of the form

$$
\frac{A(z)}{B(z)},
$$

where $A \in \mathbb{C}_{N-1}[z], B \in \mathbb{C}_{N}[z], 0 \leq \operatorname{deg}(A) \leq N-1, \operatorname{deg}(B)=N, B(0)=1, B(z) \neq 0$, for all $z \in \mathbb{C}_{+} \cup \mathbb{R}$, and $A$ and $B$ have no common factors.

Theorem 2.1. A function $u$ belongs to $M(N)$ if and only if the Hankel operator $H_{u}$ has complex rank $N$. Moreover, if $u \in \mathcal{M}(N)$ is of the form $u(z)=A(z) / B(z)$, where $B(z)=\prod_{j=1}^{J}\left(z-p_{j}\right)^{m_{j}}$ with $\sum_{j=1}^{J} m_{j}=N$ and $\operatorname{Im} p_{j}<0$ for all $j=1,2, \ldots, J$, then the range of $H_{u}$ is given by

$$
\operatorname{Ran} H_{u}=\operatorname{span}_{\mathbb{C}}\left\{\frac{1}{\left(z-p_{j}\right)^{m}} ; 1 \leq m \leq m_{j}\right\}_{j=1}^{J}
$$

Proof. The theorem will follow from two implications:

(i) $u \in M(N) \Longrightarrow \operatorname{rk}\left(H_{u}\right) \leq N$.

(ii) $\operatorname{rk}\left(H_{u}\right)=N \Longrightarrow u \in M(N)$.

Let us first prove (i). Let $u \in M(N)$, i.e., $u$ is a linear combination of terms $\frac{1}{(z-p)^{m}}$, where $\operatorname{Im} p<0$, $1 \leq m \leq m_{p}$, and $\sum m_{p}=N$. Computing the integral

$$
\int_{\mathbb{R}} \frac{e^{-i x \xi}}{(x-p)^{m}} d x
$$

using the residue theorem, we obtain that $\hat{u}(\xi)=0$ for all $\xi \leq 0$ and $\hat{u}(\xi)$ is a linear combination of terms $\xi^{m-1} e^{-i p \xi}$, with $1 \leq m \leq m_{p}$, for $\xi>0$.

Given $h \in L_{+}^{2}$, we have $\widehat{H_{u}(h)}(\xi)=0$ for $\xi<0$. For $\xi>0$, we have

$$
\begin{aligned}
\widehat{H_{u}(h)}(\xi)= & \frac{1}{2 \pi} \int_{-\infty}^{0} \hat{u}(\xi-\eta) \hat{\bar{h}}(\eta) d \eta=\frac{1}{2 \pi} \int_{0}^{\infty} \hat{u}(\xi+\eta) \overline{\hat{h}}(\eta) d \eta \\
= & \sum_{\substack{1 \leq m \leq m_{p} \\
\sum m_{p}=N}} c_{m, p}\left(\sum_{k=0}^{m-1} C_{m-1}^{k} \xi^{m-1-k} \int_{0}^{\infty} \eta^{k} \overline{\hat{h}}(\eta) e^{-i p \eta} d \eta\right) e^{-i p \xi} \\
= & \sum_{\substack{1 \leq m \leq m_{p} \\
\sum m_{p}=N}} \tilde{d}_{m, p}(u, h) \xi^{m-1} e^{-i p \xi}=\sum_{\substack{1 \leq m \leq m_{p} \\
\sum m_{p}=N}} d_{m, p}(u, h)\left(\frac{1}{(x-p)^{m}}\right)^{\wedge}
\end{aligned}
$$


where $c_{m, p}, \tilde{d}_{m, p}, d_{m, p}$ are constants depending on $p$ and $m$. Hence,

$$
H_{u}(h)(x)=\sum_{\substack{1 \leq m \leq m_{p} \\ \sum m_{p}=N}} \frac{d_{m, p}(u, h)}{(x-p)^{m}}
$$

and $\operatorname{rk}\left(H_{u}\right) \leq N$.

Let us now prove (ii). Assume that $\operatorname{rank}\left(H_{u}\right)=N$, so the range of $H_{u}$, $\operatorname{Ran} H_{u}$, is a $2 N$-dimensional real vector space. As $H_{u}$ is $\mathbb{C}$-antilinear, one can choose a basis of Ran $H_{u}$ of eigenvectors of $H_{u}$ in the following way:

$$
\left\{v_{1}, i v_{1}, \ldots, v_{N}, i v_{N} ; H_{u}\left(v_{j}\right)=\lambda_{j} v_{j}, \lambda_{j}>0, j=1,2, \ldots, N\right\}
$$

Let $w_{j}=\sqrt{\lambda}_{j} v_{j}$. If $h \in L_{+}^{2}$, then by Parseval's identity we have

$$
\begin{aligned}
H_{u}(h) & =\sum_{j=1}^{N}\left(H_{u}(h), v_{j}\right) v_{j}+\sum_{j=1}^{N}\left(H_{u}(h), i v_{j}\right) i v_{j}=2 \sum_{j=1}^{N}\left(H_{u}(h), v_{j}\right) v_{j}=2 \sum_{j=1}^{N}\left(H_{u}\left(v_{j}\right), h\right) v_{j} \\
& =2 \sum_{j=1}^{N}\left(\lambda_{j} v_{j}, h\right) v_{j}=2 \sum_{j=1}^{N}\left(w_{j}, h\right) w_{j}=\frac{1}{\pi} \sum_{j=1}^{N}\left(\int_{0}^{\infty} \hat{w}_{j}(\eta) \overline{\hat{h}}(\eta) d \eta\right) w_{j} .
\end{aligned}
$$

Consequently,

$$
\widehat{H_{u}(h)}(\xi)=\frac{1}{2 \pi} \mathbf{1}_{\xi \geq 0} \int_{0}^{\infty} \hat{u}(\xi+\eta) \overline{\hat{h}}(\eta) d \eta=\frac{1}{\pi} \mathbf{1}_{\xi \geq 0} \sum_{j=1}^{N} \int_{0}^{\infty} \hat{w}_{j}(\eta) \hat{w}_{j}(\xi) \overline{\hat{h}}(\eta) d \eta,
$$

and hence,

$$
\mathbf{1}_{\xi \geq 0} \int_{0}^{\infty}\left(\hat{u}(\xi+\eta)-2 \sum_{j=1}^{N} \hat{w}_{j}(\eta) \hat{w}_{j}(\xi)\right) \overline{\hat{h}}(\eta) d \eta=0,
$$

for all $h \in L_{+}^{2}$. Therefore, for all $\xi, \eta \geq 0$, we have

$$
\hat{u}(\xi+\eta)=2 \sum_{j=1}^{N} \hat{w}_{j}(\eta) \hat{w}_{j}(\xi)
$$

Let $L>2 N+1$ be an even integer and let $\phi$ be the probability density function of the chi-square distribution defined by

$$
\phi(\xi)= \begin{cases}2^{-L / 2} \Gamma\left(\frac{L}{2}\right)^{-1} \xi^{(L / 2)-1} e^{-\xi / 2} & \text { if } \xi \geq 0, \\ 0 & \text { if } \xi<0,\end{cases}
$$

where $\Gamma$ is the gamma function. Then, its Fourier transform is

$$
\widehat{\phi}(x)=(1+2 i x)^{-L / 2} .
$$


Notice that $\phi \in H^{N}(\mathbb{R})$ since

$$
\|\phi\|_{H^{N}}^{2}=\int_{\mathbb{R}} \frac{\langle x\rangle^{2 N}}{|1+2 i x|^{L}} d x,
$$

which is convergent if and only if $2 N-L<-1$.

Let $\langle\theta, \psi\rangle=\int_{\mathbb{R}} \theta(x) \psi(x)$ for all $\theta \in H^{-N}(\mathbb{R})$ and $\psi \in H^{N}(\mathbb{R})$. Consider the matrix $A_{\phi}$ defined by

$$
\left(\begin{array}{cccc}
\left\langle\hat{w}_{1}, \phi\right\rangle & \left\langle\hat{w}_{1}^{\prime}, \phi\right\rangle & \cdots & \left\langle\hat{w}_{1}^{(N)}, \phi\right\rangle \\
\left\langle\hat{w}_{2}, \phi\right\rangle & \left\langle\hat{w}_{2}^{\prime}, \phi\right\rangle & \cdots & \left\langle\hat{w}_{2}^{(N)}, \phi\right\rangle \\
\vdots & \vdots & \ddots & \vdots \\
\left\langle\hat{w}_{N}, \phi\right\rangle & \left\langle\hat{w}_{N}^{\prime}, \phi\right\rangle & \cdots & \left\langle\hat{w}_{N}^{(N)}, \phi\right\rangle
\end{array}\right)
$$

Since $\operatorname{rk}\left(A_{\phi}\right) \leq N$, it results that there exists $\left(c_{0}, c_{1}, \ldots, c_{N}\right) \neq 0$ such that

$$
\left\langle\sum_{k=0}^{N} c_{k} \hat{w}_{j}^{(k)}, \phi\right\rangle=0
$$

for all $j=1,2, \ldots, N$. Then, since $\operatorname{supp} \phi \subset[0, \infty)$ and by $(2-3)$, we have for all $\eta \geq 0$ that

$$
\begin{aligned}
\sum_{k=0}^{N}\left\langle c_{k} \hat{u}^{(k)}(\xi), \phi(\xi-\eta)\right\rangle_{\xi} & =\sum_{k=0}^{N}\left\langle c_{k} \hat{u}^{(k)}(\xi+\eta), \phi(\xi)\right\rangle_{\xi}=\sum_{k=0}^{N}(-1)^{k} c_{k} \int_{0}^{\infty} \hat{u}(\xi+\eta) \phi^{(k)}(\xi) d \xi \\
& =2 \sum_{k=0}^{N}(-1)^{k} c_{k} \int_{0}^{\infty}\left(\sum_{j=1}^{N} \hat{w}_{j}(\eta) \hat{w}_{j}(\xi)\right) \phi^{(k)}(\xi) d \xi \\
& =2 \sum_{j=1}^{N} \hat{w}_{j}(\eta) \sum_{k=0}^{N} c_{k}\left\langle\hat{w}_{j}^{(k)}(\xi), \phi(\xi)\right\rangle=0 .
\end{aligned}
$$

Set $T=\sum_{k=0}^{N} c_{k} \hat{u}^{(k)}$. Then $T \in H^{-N}$ and $\operatorname{supp} T \in[0, \infty)$. We have just proved that for all $\eta \geq 0$

$$
\begin{aligned}
0 & =\langle T, \phi(\cdot-\eta)\rangle=\int_{\mathbb{R}} T(\xi) \phi(\xi-\eta) d \xi=\int_{\mathbb{R}} T(\xi)\left(\int_{\mathbb{R}} \frac{e^{i x(\xi-\eta)}}{(1+2 i x)^{L / 2}} d x\right) d \xi \\
& =\int_{\mathbb{R}}\left(\int_{\mathbb{R}} T(\xi) e^{i x \xi} d \xi\right) \frac{e^{-i x \eta}}{(1+2 i x)^{L / 2}} d x=\int_{\mathbb{R}} \mathscr{F}^{-1} T(x) \frac{e^{-i x \eta}}{(1+2 i x)^{L / 2}} d x .
\end{aligned}
$$

Seting $R(x):=\frac{1}{(1+2 i x)^{L / 2}} \mathscr{F}^{-1} T(x)$, we have $\hat{R} \in H^{L / 2-N}(\mathbb{R}) \subset H^{1 / 2}(\mathbb{R})$ and

$$
0=\int_{\mathbb{R}} R(x) e^{-i x \eta} d x=\hat{R}(\eta) \quad \text { for all } \eta \geq 0 .
$$

Thus supp $\hat{R} \subset(-\infty, 0]$. By the definition of $R,\left(1-2 D_{\xi}\right)^{L / 2} \hat{R}(\xi)=T(\xi)$. Since the left hand-side is supported on $(-\infty, 0]$ and the right hand-side is supported on $[0, \infty)$, we deduce that $\operatorname{supp} T \subset 0$. In particular, $T_{\mid \xi>0}=0$. This yields that $\hat{u}_{\mid \xi>0}$ is a weak solution on $(0, \infty)$ of the linear ordinary differential 
equation

$$
\sum_{k=0}^{N} c_{k} v^{(k)}(\xi)=0
$$

Then, by [Hörmander 1990, Theorem 4.4.8, p. 115], we have $\hat{u}_{\mid \xi>0} \in C^{N}((0, \infty))$; further, $\hat{u}_{\mid \xi>0}$ is a classical solution of this equation, and therefore it is a linear combination of terms

$$
\xi^{m-1} e^{q \xi}
$$

where $q \in \mathbb{C}$ is a root of the polynomial $P(X)=\sum_{k=0}^{N} c_{k} X^{k}$ with multiplicity $m_{q}, 1 \leq m \leq m_{q}$, and $\sum_{q} m_{q}=N$. Note that we must have $\operatorname{Re} q<0$, because $u \in L_{+}^{2}(\mathbb{R})$. Therefore we will set $q=-i p$, with $\operatorname{Im} p<0$, and obtain that $\hat{u}(\xi)$ is a linear combination of terms $\xi^{m-1} e^{-i p \xi}$ for $\xi>0$. By the hypothesis $u \in L_{+}^{2}(\mathbb{R})$, we obtain $\hat{u}(\xi)=0$ for $\xi \leq 0$. Hence, for all $\xi \in \mathbb{R}, \hat{u}(\xi)$ is a linear combination of $\left((x-p)^{-m}\right)^{\wedge}(\xi)$, with $1 \leq q \leq m_{q}$ and $\sum m_{q}=N$. Thus $u \in M\left(N^{\prime}\right)$ for some $N^{\prime} \leq N$. If $N^{\prime}<N$, implication (i) above yields $\operatorname{rk}\left(H_{u}\right) \leq N^{\prime}$, contradicting our assumption. In conclusion, $u \in M(N)$.

Finally, when $u \in M(N)$ we have $\operatorname{rk}\left(H_{u}\right)=N$ and (2-2), and thus (2-1) follows.

As a consequence of (2-1) we make the following remark.

Remark 2.2. If $u \in M(N)$, then $u \in \operatorname{Ran} H_{u}$.

\section{Spectral properties of the operator $A_{u}$ for a traveling wave $u$}

Let us first recall the definition and the basic properties of the generalized wave operators, which are the main objects in scattering theory. We refer to [Reed and Simon 1979, Chapter XI] for more details.

Let $A$ and $B$ be two self-adjoint operators on a Hilbert space $\mathscr{H}$. The basic principle of scattering theory is to compare the free dynamics corresponding to $e^{-i A t}$ and $e^{-i B t}$. The fact that $e^{-i B t} \phi$ "looks asymptotically free" as $t \rightarrow-\infty$, with respect to A, means that there exists $\phi_{+} \in \mathscr{H}$ such that

$$
\lim _{t \rightarrow-\infty}\left\|e^{-i B t} \phi-e^{-i t A} \phi_{+}\right\|=0
$$

or, equivalently,

$$
\lim _{t \rightarrow-\infty}\left\|e^{i A t} e^{-i t B} \phi-\phi_{+}\right\|=0 .
$$

Hence, we reduced ourselves to the problem of the existence of a strong limit. Let $\mathscr{H}_{\mathrm{ac}}(B)$ be the absolutely continuous subspace for $B$ and let $P_{\text {ac }}(B)$ be the orthogonal projection onto this subspace. In the definition of the generalized wave operators we have $\phi \in \mathscr{H}_{\mathrm{ac}}(B)$.

We say that the generalized wave operators exist if the following strong limits exist:

$$
\Omega^{ \pm}(A, B)=\lim _{t \rightarrow \mp \infty} e^{i t A} e^{-i t B} P_{\mathrm{ac}}(B)
$$

The wave operators $\Omega^{ \pm}(A, B)$ are partial isometries with initial subspace $\mathscr{H}_{\mathrm{ac}}(B)$ and with values in Ran $\Omega^{ \pm}(A, B)$. Moreover, $\operatorname{Ran} \Omega^{ \pm}(A, B) \subset \mathscr{H}_{\mathrm{ac}}(A)$. If $\operatorname{Ran} \Omega^{ \pm}(A, B)=\mathscr{H}_{\mathrm{ac}}(A)$, we say that the generalized wave operators are complete. Lastly, we note that

$$
A \Omega^{ \pm}(A, B)=\Omega^{ \pm}(A, B) B .
$$


Lemma 3.1. If $u \in H_{+}^{1 / 2}$ is a traveling wave, then $u \in H_{+}^{s}(\mathbb{R})$ for all $s \geq 0$. In particular, by Sobolev embedding theorem, we have $u \in L^{p}(\mathbb{R})$ for $2 \leq p \leq \infty$.

Proof. Because $u \in H^{1 / 2}(\mathbb{R})$, the Sobolev embedding theorem yields $u \in L^{p}(\mathbb{R})$, for all $2 \leq p<\infty$. Therefore $|u|^{2} u \in L^{2}(\mathbb{R})$ and thus $\Pi\left(|u|^{2} u\right) \in L_{+}^{2}$. Using equation (1-4), namely

$$
c D u+\omega u=\Pi\left(|u|^{2} u\right),
$$

we deduce that $D u \in L_{+}^{2}$. Consequently, $u \in H_{+}^{1}$ and by Sobolev embedding theorem we have $u \in L^{\infty}(\mathbb{R})$. Then $u^{2} D \bar{u},|u|^{2} D u \in L^{2}(\mathbb{R})$. Applying the operator $D$ to both sides of (1-4), we obtain $D^{2} u \in L^{2}(\mathbb{R})$ and hence $u \in H_{+}^{2}$. Iterating this argument infinitely many times, the conclusion follows.

Proposition 3.2. Let $u$ be a traveling wave. Then, $\left(A_{u}+i\right)^{-1}-(D+i)^{-1}$ is a trace class operator. Proof. We prove first that for all $f \in L^{2}(\mathbb{R})$, the operator $(D+i)^{-1} f$, defined on $L^{2}(\mathbb{R})$ by

$$
\left((D+i)^{-1} f\right) h(x)=(D+i)^{-1}(f h)(x)
$$

is Hilbert-Schmidt. Denote by $\mathscr{F}$ the Fourier transform. In view of the isomorphism of $L^{2}(\mathbb{R})$ induced by the Fourier transform, $(D+i)^{-1} f$ is a Hilbert-Schmidt operator if and only if $\mathscr{F}(D+i)^{-1} f$ is one. The latter is an integral operator of kernel

$$
K(\xi, \eta)=\frac{1}{2 \pi} \cdot \frac{1}{\xi+i} \hat{f}(\xi-\eta) .
$$

Indeed,

$$
\mathscr{F}\left((D+i)^{-1} f h\right)(\xi)=\frac{1}{2 \pi} \cdot \frac{1}{\xi+i} \widehat{f h}(\xi)=\frac{1}{2 \pi} \int_{\mathbb{R}} \frac{1}{\xi+i} \hat{f}(\xi-\eta) \hat{h}(\eta) d \eta=\int_{\mathbb{R}} K(\xi, \eta) \hat{h}(\eta) d \eta .
$$

Therefore, it is Hilbert-Schmidt if and only if $K(\xi, \eta) \in L_{\xi, \eta}^{2}(\mathbb{R} \times \mathbb{R})$. By the change of variables $\eta \mapsto \zeta=\xi-\eta$ we have

$$
\|K(\xi, \eta)\|_{L_{\xi, \eta}^{2}}^{2}=\frac{1}{4 \pi^{2}} \int_{\mathbb{R}} \frac{d \xi}{\xi^{2}+1} \int_{\mathbb{R}}|\hat{f}(\zeta)|^{2} d \zeta=C\|f\|_{L^{2}}^{2}<\infty .
$$

Hence $(D+i)^{-1} f$ is a Hilbert-Schmidt operator and so is $\bar{f}(D+i)^{-1}$, its adjoint. According to Lemma 3.1, $u \in L^{\infty}(\mathbb{R})$ and thus $|u|^{2} \in L^{2}(\mathbb{R})$. Taking $f=|u|^{2}$ and $f=u$, we conclude that the operators $(D+i)^{-1}|u|^{2},(D+i)^{-1} u$, and $\bar{u}(D+i)^{-1}$ are all Hilbert-Schmidt.

We write

$$
\begin{aligned}
\left(A_{u}+i\right)^{-1}-(D+i)^{-1} & =(D+i)^{-1}\left(D-A_{u}\right)\left(A_{u}+i\right)^{-1} \\
& =\frac{1}{c}(D+i)^{-1} T_{|u|^{2}}\left(A_{u}+i\right)^{-1} \\
& =\frac{1}{c} \Pi(D+i)^{-1}|u|^{2}\left(A_{u}+i\right)^{-1}=L\left(A_{u}+i\right)^{-1},
\end{aligned}
$$

where $L=\frac{1}{c} \Pi(D+i)^{-1}|u|^{2}$. Note that $L$ is a Hilbert-Schmidt operator since it is the composition of the bounded operator $\frac{1}{c} \Pi: L^{2}(\mathbb{R}) \rightarrow L_{+}^{2}$ with the Hilbert-Schmidt operator $(D+i)^{-1}|u|^{2}$. Finally, we 
write, using the latter formula twice

$$
\begin{aligned}
\left(A_{u}+i\right)^{-1}-(D+i)^{-1} & =L\left(L\left(A_{u}+i\right)^{-1}+(D+i)^{-1}\right) \\
& =L \circ L \circ\left(A_{u}+i\right)^{-1}+\frac{1}{c} \Pi(D+i)^{-1} u \circ \bar{u}(D+i)^{-1} .
\end{aligned}
$$

We obtain that $\left(A_{u}+i\right)^{-1}-(D+i)^{-1}$ is a trace class operator since the composition of two HilbertSchmidt operators is a trace class operator.

Corollary 3.3. If $u$ is a traveling wave, the wave operators $\Omega^{ \pm}\left(D, A_{u}\right)$ exist and are complete.

Proof. This easily follows from Kuroda-Birman theorem [Reed and Simon 1979, Theorem XI.9]: Let A and $B$ be two self-adjoint operators on a Hilbert space such that $(A+i)^{-1}-(B+i)^{-1}$ is a trace class operator. Then $\Omega^{ \pm}(A, B)$ exist and are complete.

Corollary 3.4. If $u$ is a traveling wave, then $\sigma_{\mathrm{ac}}\left(A_{u}\right)=[0,+\infty)$.

Proof. Since $\Omega^{ \pm}\left(D, A_{u}\right)$ are complete, it results that they are isometries from $\mathscr{H}_{\mathrm{ac}}\left(A_{u}\right)$ onto $\mathscr{H}_{\mathrm{ac}}(D)=L_{+}^{2}$. By (3-2), we then have

$$
A_{\left.u\right|_{\mathscr{H}_{\mathrm{ac}}\left(A_{u}\right)}}=\left[\Omega^{ \pm}\left(D, A_{u}\right)_{\mathscr{H}_{\mathrm{ac}}\left(A_{u}\right)}\right]^{-1} D \Omega^{ \pm}\left(D, A_{u}\right)_{\mathscr{H}_{\mathrm{ac}}\left(A_{u}\right)} .
$$

Consequently, $\sigma_{\mathrm{ac}}\left(A_{u}\right)=\sigma_{\mathrm{ac}}(D)=[0,+\infty)$.

Our main goal in the following is to prove that $\mathscr{H}_{\mathrm{ac}}\left(A_{u}\right) \subset \operatorname{Ker} H_{u}$. As we will see below, it is enough to prove that $\left[\Omega^{+}\left(D, A_{u}\right) H_{u}^{2}\right]\left(\mathscr{H}_{\mathrm{ac}}\left(A_{u}\right)\right)=0$.

Lemma 3.5. $H_{u}$ is a Hilbert-Schmidt operator on $L_{+}^{2}(\mathbb{R})$ of Hilbert-Schmidt norm $\frac{1}{\sqrt{2 \pi}}\|u\|_{\dot{H}_{+}^{1 / 2}}$.

Proof. Denote by $\|T\|_{H S}$ the Hilbert-Schmidt norm of a Hilbert-Schmidt operator $T$. By (2-2), we have

$$
\widehat{H_{u}(h)}(\xi)=\frac{1}{2 \pi} \mathbf{1}_{\xi \geq 0} \int_{0}^{\infty} \hat{u}(\xi+\eta) \overline{\hat{h}}(\eta) d \eta .
$$

Then, we obtain

$$
\begin{aligned}
H_{u}(h)(x) & =\frac{1}{4 \pi^{2}} \int_{0}^{\infty} \int_{0}^{\infty} e^{i x \xi} \hat{u}(\xi+\eta) \overline{\hat{h}}(\eta) d \eta d \xi \\
& =\frac{1}{4 \pi^{2}} \int_{\mathbb{R}}\left(\int_{0}^{\infty} \int_{0}^{\infty} e^{i x \xi} e^{i y \eta} \hat{u}(\xi+\eta) d \eta d \xi\right) \bar{h}(y) d y
\end{aligned}
$$

Using the fact that the Hilbert-Schmidt norm of an operator is equal to the norm of its integral kernel, Plancherel's formula, and Fubini's theorem, we have

$$
\begin{aligned}
\left\|H_{u}(h)\right\|_{H S}^{2} & =\frac{1}{16 \pi^{4}}\left\|\int_{0}^{\infty} \int_{0}^{\infty} e^{i x \xi} e^{i y \eta} \hat{u}(\xi+\eta) d \eta d \xi\right\|_{L_{x, y}^{2}}^{2}=\frac{1}{4 \pi^{2}}\left\|\mathbf{1}_{\xi \geq 0} \mathbf{1}_{\eta \geq 0} \hat{u}(\xi+\eta)\right\|_{L_{\eta, \xi}^{2}}^{2} \\
& =\frac{1}{4 \pi^{2}} \int_{0}^{\infty} \int_{0}^{\infty}|\hat{u}(\xi+\eta)|^{2} d \eta d \xi=\frac{1}{4 \pi^{2}} \int_{0}^{\infty} \int_{\xi}^{\infty}|\hat{u}(\zeta)|^{2} d \zeta d \xi \\
& =\frac{1}{4 \pi^{2}} \int_{0}^{\infty}\left(\int_{0}^{\zeta} d \xi\right)|\hat{u}(\zeta)|^{2} d \zeta=\frac{1}{4 \pi^{2}} \int_{0}^{\infty} \zeta|\hat{u}(\zeta)|^{2} d \zeta=\frac{1}{2 \pi}\|u\|_{\dot{H}^{1 / 2}}^{2}
\end{aligned}
$$


Lemma 3.6. $\operatorname{Ker} H_{u}^{2}=\operatorname{Ker} H_{u}$. Moreover, if $\operatorname{Ran} H_{u}$ is finite-dimensional, then $\operatorname{Ran} H_{u}^{2}=\operatorname{Ran} H_{u}$. Proof. Let $f \in \operatorname{Ker} H_{u}^{2}$. Then, by (1-7) and the fact that $\left(H_{u}\left(h_{1}\right), h_{2}\right)=\left(H_{u}\left(h_{2}\right), h_{1}\right)$ for all $h_{1}, h_{2} \in L_{+}^{2}$, we have

$$
\left\|H_{u} f\right\|_{L^{2}}^{2}=\left(H_{u} f, H_{u} f\right)=\left(H_{u}^{2} f, f\right)=0,
$$

and thus $H_{u} f=0$. Hence, $\operatorname{Ker} H_{u}^{2} \subset \operatorname{Ker} H_{u}$. Therefore, we obtain $\operatorname{Ker} H_{u}^{2}=\operatorname{Ker} H_{u}$ since the inverse inclusion is obvious.

The identity (1-7) yields also Ker $H_{u}=\left(\operatorname{Ran} H_{u}\right)^{\perp}$. Moreover, it implies that $H_{u}^{2}$ is a self-adjoint operator and therefore, $\operatorname{Ker} H_{u}^{2}=\left(\operatorname{Ran} H_{u}^{2}\right)^{\perp}$. Hence, we obtain

$$
\left(\operatorname{Ran} H_{u}^{2}\right)^{\perp}=\left(\operatorname{Ran} H_{u}\right)^{\perp} .
$$

Taking the orthogonal complement of both sides, this yields

$$
\overline{\operatorname{Ran} H_{u}^{2}}=\overline{\operatorname{Ran} H_{u}} \text {. }
$$

If Ran $H_{u}$ is finite-dimensional, so is $\operatorname{Ran} H_{u}^{2}$, since $\operatorname{Ran} H_{u}^{2} \subset \operatorname{Ran} H_{u}$. Thus, Ran $H_{u}^{2}$ and $\operatorname{Ran} H_{u}$ are closed. It follows that $\operatorname{Ran} H_{u}^{2}=\operatorname{Ran} H_{u}$.

Lemma 3.7. If $u$ is a traveling wave, then

$$
A_{u} H_{u}^{2}=H_{u}^{2} A_{u} .
$$

Consequently, if $\operatorname{Ran} H_{u}$ is finite-dimensional, then $A_{u}\left(\operatorname{Ran} H_{u}\right) \subset \operatorname{Ran} H_{u}$.

Proof. The commutativity relation (3-3) is a consequence of identity (1-10). The second statement then follows by Lemma 3.6, $\operatorname{Ran} H_{u}^{2}=\operatorname{Ran} H_{u}$.

It is a classical fact that if $A$ and $B$ are two self-adjoint operators on a Hilbert space $\mathscr{H}$ such that $A B=B A$, then $B\left(\mathscr{H}_{\mathrm{ac}}(A)\right) \subset \mathscr{H}_{\mathrm{ac}}(A)$. For completeness, we prove this here in the case of $A_{u}$ and $H_{u}^{2}$ :

\section{Lemma 3.8.}

$$
H_{u}^{2} \mathscr{H}_{\mathrm{ac}}\left(A_{u}\right) \subset \mathscr{H}_{\mathrm{ac}}\left(A_{u}\right)
$$

Proof. As we will see below, the inclusion follows if we prove that $\mu_{H_{u}^{2} \phi} \ll \mu_{\phi}$ for all $\phi \in L_{+}^{2}$, where the $\mu_{H_{u}^{2} \phi}$ and $\mu_{\phi}$ denote the spectral measures of $H_{u}^{2} \phi$ and $\phi$ with respect to the operator $A_{u}$.

Let $E \subset \mathbb{R}$ be a measurable set and $f=\mathbf{1}_{E}$. By (3-3) and the Cauchy-Schwarz inequality we have

$$
\begin{aligned}
\mu_{H_{u}^{2} \phi}(E) & =\int_{\mathbb{R}} f d \mu_{H_{u}^{2} \phi}=\left(H_{u}^{2} \phi, f\left(A_{u}\right) H_{u}^{2} \phi\right)=\left(H_{u}^{2} \phi, H_{u}^{2} f\left(A_{u}\right) \phi\right)=\left(H_{u}^{4} \phi, f\left(A_{u}\right) \phi\right) \\
& \leq \sqrt{\left(f\left(A_{u}\right) \phi, f\left(A_{u}\right) \phi\right)}\left\|H_{u}^{4} \phi\right\|_{L^{2}}=\sqrt{\left(\phi, f\left(A_{u}\right) \phi\right)}\left\|H_{u}^{4} \phi\right\|_{L^{2}}=\sqrt{\mu_{\phi}(E)}\left\|H_{u}^{4} \phi\right\|_{L^{2}} .
\end{aligned}
$$

Therefore, $\mu_{H_{u}^{2} \phi} \ll \mu_{\phi}$. Denote by $m$ the Lebesgue measure on $\mathbb{R}$. If $\phi \in \mathscr{H}_{\mathrm{ac}}\left(A_{u}\right)$, then $\mu_{\phi} \ll m$ and thus $\mu_{H_{u}^{2} \phi} \ll m$. Hence, $H_{u}^{2} \mathscr{H}_{\mathrm{ac}}\left(A_{u}\right) \subset \mathscr{H}_{\mathrm{ac}}\left(A_{u}\right)$.

Proposition 3.9. If $u$ is a traveling wave, then $\mathscr{H}_{\mathrm{ac}}\left(A_{u}\right) \subset \operatorname{Ker} H_{u}$.

Proof. It is enough to prove that $\left[\Omega^{+}\left(D, A_{u}\right) H_{u}^{2}\right]\left(\mathscr{H}_{\mathrm{ac}}\left(A_{u}\right)\right)=0$. If this holds, then $H_{u}^{2}\left(\mathscr{H}_{\mathrm{ac}}\left(A_{u}\right)\right)=0$ since $H_{u}^{2} \mathscr{H}_{\mathrm{ac}}\left(A_{u}\right) \subset \mathscr{H}_{\mathrm{ac}}\left(A_{u}\right)$ and $\Omega^{+}\left(D, A_{u}\right)$ is an isometry on $\mathscr{H}_{\mathrm{ac}}\left(A_{u}\right)$. Therefore, $\mathscr{H}_{\mathrm{ac}}\left(A_{u}\right) \subset$ $\operatorname{Ker} H_{u}^{2}=\operatorname{Ker} H_{u}$. 
First note that

$$
H_{u} e^{i t D}=e^{i t D} H_{\tau_{t}(u)}
$$

where $\tau_{a}$ denotes the translation $\tau_{a} u(x)=u(x-a)$. Indeed, for $f \in L_{+}^{2}$, passing into Fourier space, we have

$$
\begin{aligned}
\left(H_{u} e^{i t D} f\right)^{\wedge}(\xi) & =\mathbf{1}_{\xi \geq 0}\left(u \overline{e^{i t D} f}\right)^{\wedge}(\xi)=\frac{1}{2 \pi} \mathbf{1}_{\xi \geq 0} \int_{\mathbb{R}} \hat{u}(\xi-\eta) e^{i t \eta} \hat{\bar{f}}(\eta) d \eta \\
& =\frac{1}{2 \pi} \mathbf{1}_{\xi \geq 0} e^{i t \xi} \int e^{-i t(\xi-\eta)} \hat{u}(\xi-\eta) \hat{\bar{f}}(\eta) d \eta=\mathbf{1}_{\xi \geq 0} e^{i t \xi}\left(\tau_{t}(u) \bar{f}\right)^{\wedge}(\xi) \\
& =\mathbf{1}_{\xi \geq 0}\left(e^{i t D}\left(\tau_{t}(u) \bar{f}\right)\right)^{\wedge}(\xi)=\left(e^{i t D} H_{\tau_{t}(u)} f\right)^{\wedge}(\xi) .
\end{aligned}
$$

By Lemma 3.8, (3-3), and (3-4), we have for all $f \in \mathscr{H}_{\mathrm{ac}}\left(A_{u}\right)$

$$
\begin{aligned}
e^{i t D} e^{-i t A_{u}} P_{\mathrm{ac}} H_{u}^{2} f & =e^{i t D} e^{-i t A_{u}} H_{u}^{2} f=e^{i t D} H_{u}^{2} e^{-i t A_{u}} f=e^{i t D} H_{u} H_{u} e^{-i t D} e^{i t D} e^{-i t A_{u}} f \\
& =e^{i t D} H_{u} e^{-i t D} H_{\tau_{-t}(u)} e^{i t D} e^{-i t A_{u}} f=H_{\tau_{-t}(u)}^{2} e^{i t D} e^{-i t A_{u}} P_{\mathrm{ac}}\left(A_{u}\right) f
\end{aligned}
$$

We intend to prove that $H_{\tau_{-t}(u)}^{2} e^{i t D} e^{-i t A_{u}} P_{\mathrm{ac}}\left(A_{u}\right) f$ tends to 0 in the $L_{+}^{2}$-norm as $t \rightarrow-\infty$. From this, we conclude that $\Omega^{+}\left(D, A_{u}\right) H_{u}^{2} f=0$. Since, by Lemma 3.5, $H_{\tau_{-t}(u)}$ is uniformly bounded, it suffices to prove that $H_{\tau_{-t}(u)} e^{i t D} e^{-i t A_{u}} P_{\mathrm{ac}}\left(A_{u}\right) f$ tends to 0 . We have

$$
\begin{aligned}
& \left\|H_{\tau_{-t}(u)} e^{i t D} e^{-i t A_{u}} P_{\mathrm{ac}}\left(A_{u}\right) f\right\|_{L_{+}^{2}} \\
& \quad \leq\left\|H_{\tau_{-t}(u)}\left(e^{i t D} e^{-i t A_{u}} P_{\mathrm{ac}}\left(A_{u}\right) f-\Omega^{+}\left(D, A_{u}\right) f\right)\right\|_{L_{+}^{2}}+\left\|H_{\tau_{-t}(u)} \Omega^{+}\left(D, A_{u}\right) f\right\|_{L_{+}^{2}} \\
& \quad \leq \frac{1}{\sqrt{2 \pi}}\|u\|_{\dot{H}^{1 / 2}}\left\|e^{i t D} e^{-i t A_{u}} P_{\mathrm{ac}}\left(A_{u}\right) f-\Omega^{+}\left(D, A_{u}\right) f\right\|_{L_{+}^{2}}+\int_{\mathbb{R}}|u(x+t)|^{2}\left|\Omega^{+}\left(D, A_{u}\right) f(x)\right|^{2} d x
\end{aligned}
$$

The first term on the last line converges to 0 by the definition of the wave operator $\Omega^{+}\left(D, A_{u}\right)$. Since $u$ is a traveling wave, we can write

$$
u \in \bigcap_{s \geq 0} H^{s}(\mathbb{R}) \subset C_{\rightarrow 0}^{\infty}(\mathbb{R}),
$$

where $C_{\rightarrow 0}^{\infty}(\mathbb{R})$ is the space of functions $f$ of class $C^{\infty}$ such that $\lim _{x \rightarrow-\infty} D^{k} f(x)=\lim _{x \rightarrow \infty} D^{k} f(x)=0$ for all $k \in \mathbb{N}$. Therefore, for arbitrary fixed $x$, we have

$$
\lim _{t \rightarrow-\infty} \tau_{-t}(u)(x)=\lim _{t \rightarrow-\infty} u(x+t)=0
$$

Note also that

$$
|u(x+t)|^{2}\left|\Omega^{+}\left(D, A_{u}\right) f(x)\right|^{2} \leq\|u\|_{L^{\infty}}^{2}\left|\Omega^{+}\left(D, A_{u}\right) f(x)\right|^{2}
$$

for all $x \in \mathbb{R}$. Thus the last term in (3-5) converges to 0 by the dominated convergence theorem. This shows that $\left[\Omega^{+}\left(D, A_{u}\right) H_{u}^{2}\right]\left(\mathcal{H}_{\mathrm{ac}}\left(A_{u}\right)\right)=0$. 


\section{Classification of traveling waves}

Lemma 4.1. There are no nontrivial traveling waves of velocity $c=0$ in $L_{+}^{2}(\mathbb{R})$.

Proof. Let $u$ be a nontrivial traveling wave of velocity $c=0$. Then, (1-4) gives $\Pi\left(|u|^{2} u\right)=\omega u$. Taking the scalar product with $e^{i \xi x} u(x)$, where $\xi \geq 0$, we obtain

$$
\mathscr{F}\left(|u|^{4}-\omega|u|^{2}\right)(\xi)=0,
$$

where $\mathscr{F}$ denotes the Fourier transform. Since $|u|^{4}-\omega|u|^{2}$ is a real-valued function, we have that the last equality holds for all $\xi \in \mathbb{R}$. Thus $|u|^{4}-\omega|u|^{2}=0$ on $\mathbb{R}$ and therefore $u(x)=0$ or $|u(x)|^{2}=\omega>0$, for all $x \in \mathbb{R}$. Since the function $u$ is holomorphic on $\mathbb{C}_{+}$, its trace on $\mathbb{R}$ is either identically zero, or the set of zeros of $u$ on $\mathbb{R}$ has Lebesgue measure zero. In conclusion, we have $|u|^{2}=\omega>0$ a.e. on $\mathbb{R}$ and thus $u$ is not a function in $L_{+}^{2}(\mathbb{R})$.

Lemma 4.2. If $u \in H_{+}^{s}$ for $s>\frac{1}{2}$ and $v \in \operatorname{Ker} H_{u}$, then $\bar{u} v \in L_{+}^{2}$. Moreover, if $u \in L^{\infty}(\mathbb{R})$, then $T_{|u|^{2}} v=|u|^{2} v$.

Proof. Indeed, $0=H_{u}(v)=\Pi(u \bar{v})$ and thus $\bar{u} v \in L_{+}^{2}$. Furthermore, since $u, \bar{u} v \in L_{+}^{2}$, we obtain $T_{|u|^{2}} v=\Pi(u \bar{u} v)=|u|^{2} v$.

Lemma 4.3. Let $u \in H_{+}^{s}, s>\frac{1}{2}$, be a solution of the cubic Szegó equation (1-2). For the Cauchy problem

$$
\left\{\begin{array}{l}
i \partial_{t} \psi=|u(t)|^{2} \psi \\
\left.\psi\right|_{t=0}=\psi_{0}
\end{array}\right.
$$

if $\psi_{0} \in \operatorname{Ker} H_{u(0)}$, then $\psi(t) \in \operatorname{Ker} H_{u(t)}$ for all $t \in \mathbb{R}$.

Proof. Let us first consider

$$
\left\{\begin{array}{l}
i \partial_{t} \psi_{1}=T_{|u(t)|^{2}} \psi_{1} \\
\left.\psi_{1}\right|_{t=0}=\psi_{0}
\end{array}\right.
$$

Using the Lax pair structure, we have

$$
\begin{aligned}
\partial_{t} H_{u}\left(\psi_{1}\right) & =\left[B_{u}, H_{u}\right] \psi_{1}+H_{u} \partial_{t} \psi_{1}=\left[\frac{i}{2} H_{u}^{2}-i T_{|u|^{2}}, H_{u}\right] \psi_{1}+H_{u}\left(-i T_{|u|^{2}} \psi_{1}\right) \\
& =-i T_{|u|^{2}} H_{u} \psi_{1}-i H_{u} T_{|u|^{2}} \psi_{1}+i H_{u} T_{|u|^{2}} \psi_{1}=-i T_{|u|^{2}} H_{u} \psi_{1} .
\end{aligned}
$$

The solution of the linear Cauchy problem

$$
\left\{\begin{array}{l}
\partial_{t} H_{u}\left(\psi_{1}\right)=-i T_{|u|^{2}} H_{u} \psi_{1} \\
H_{u}\left(\psi_{1}(0)\right)=0
\end{array}\right.
$$

is identically zero: $H_{u(t)} \psi_{1}(t)=0$ for all $t \in \mathbb{R}$. Consequently, $\psi_{1}(t) \in \operatorname{Ker} H_{u(t)}$, and by Lemma 4.2 we obtain $T_{|u|^{2}} \psi_{1}=|u|^{2} \psi_{1}$. In conclusion, $\psi(t)=\psi_{1}(t) \in \operatorname{Ker} H_{u(t)}$.

The space $\operatorname{Ker} H_{u}$ is invariant under multiplication by $e^{i \alpha x}$, for all $\alpha \geq 0$. Indeed, suppose $f \in \operatorname{Ker} H_{u}$. Then $(u \bar{f})^{\wedge}(\xi)=0$, for all $\xi \geq 0$ and

$$
\left(H_{u}\left(e^{i \alpha x} f\right)\right)^{\wedge}(\xi)=\left(e^{-i \alpha x} u \bar{f}\right)^{\wedge}(\xi)=(u \bar{f})^{\wedge}(\xi+\alpha)=0,
$$

for all $\xi, \alpha \geq 0$. Hence, $e^{i \alpha x} f \in \operatorname{Ker} H_{u}$ for all $\alpha \geq 0$.

One can then apply the following theorem to the subspaces Ker $H_{u_{0}}$. 
Proposition 4.4 [Lax 1959]. Every nonempty closed subspace of $L_{+}^{2}$ that is invariant under multiplication by $e^{i \alpha x}$ for all $\alpha \geq 0$ is of the form $F L_{+}^{2}$, where $F$ is an analytic function in the upper-half plane, $|F(z)| \leq 1$ for all $z \in \mathbb{C}_{+}$, and $|F(x)|=1$ for all $x \in \mathbb{R}$. Moreover, $F$ is uniquely determined up to multiplication by a complex constant of absolute value 1 .

We deduce that $\operatorname{Ker} H_{u_{0}}=\phi L_{+}^{2}$, where $\phi$ is a holomorphic function in the upper half-plane $\mathbb{C}_{+}$, satisfying $|\phi(x)|=1$ on $\mathbb{R}$ and $|\phi(z)| \leq 1$ for all $z \in \mathbb{C}_{+}$.

Functions satisfying the properties in Proposition 4.4 are called inner functions in the sense of Beurling and Lax. A special class of such functions is that of Blaschke products. Given $\lambda_{j} \in \mathbb{C}$ such that for all $j$

$$
\operatorname{Im} \lambda_{j}>0 \text { and } \sum_{j} \frac{\operatorname{Im} \lambda_{j}}{1+\left|\lambda_{j}\right|^{2}}<\infty,
$$

the corresponding Blaschke product is defined by

$$
B(z)=\prod_{j} \varepsilon_{j} \frac{z-\lambda_{j}}{z-\bar{\lambda}_{j}}, \quad \text { where } \varepsilon_{j}=\frac{\left|\lambda_{j}^{2}+1\right|}{\lambda_{j}^{2}+1}
$$

(by definition $\varepsilon_{j}=1$ if $\lambda_{j}=1$ ).

Inner functions have a canonical factorization, which is analogous to the canonical factorization of inner functions on the unit disk; see [Rudin 1974, Theorem 17.15] or [Nikolski 2002, Theorem 6.4.4]. More precisely, every inner function $F$ can be written as the product

$$
F(z)=\lambda B(z) e^{i a z} \exp \left(i \int_{\mathbb{R}} \frac{1+t z}{t-z} d v(t)\right),
$$

where $z \in \mathbb{C}_{+}, \lambda \in \mathbb{C}$ with $|\lambda|=1, a \geq 0, B$ is a Blaschke product, and $v$ is a positive singular measure with respect to the Lebesgue measure. In particular, the inner function $\phi$ has such a canonical factorization.

Proposition 4.5. Let $u$ be a traveling wave and denote by $\phi$ an inner function such that $\operatorname{Ker} H_{u_{0}}=\phi L_{+}^{2}$. Then, $\phi$ satisfies the following equation on $\mathbb{R}$ :

$$
c D \phi=\left|u_{0}\right|^{2} \phi
$$

Proof. Since $u(t, x)=e^{-i \omega t} u_{0}(x-c t)$, we have $H_{u(t)}=e^{-i \omega t} \tau_{c t} H_{u_{0}} \tau_{-c t}$. Thus,

$$
\operatorname{Ker} H_{u(t)}=\tau_{c t} \operatorname{Ker} H_{u_{0}}=\tau_{c t}(\phi) L_{+}^{2} .
$$

Let $f \in L_{+}^{2}$ and let $\psi_{0}=\phi f \in \operatorname{Ker} H_{u_{0}}$ be the initial data of the Cauchy problem (4-1) in Lemma 4.3. We then have $\phi e^{-i \int_{0}^{t}|u(s)|^{2} d s} f \in \operatorname{Ker} H_{u(t)}$. Therefore,

$$
\phi e^{-i \int_{0}^{t}|u(s)|^{2} d s} L_{+}^{2} \subset \tau_{c t}(\phi) L_{+}^{2} .
$$

Conversely, by solving backward the problem (4-1) with the initial data in $\tau_{c t}(\phi) L_{+}^{2}$ at time $t$, up to the time $t=0$, we obtain

$$
\tau_{c t}(\phi) L_{+}^{2} \subset \phi e^{-i \int_{0}^{t}|u(s)|^{2} d s} L_{+}^{2}
$$

and thus, the two sets are equal. 
Let us first prove that $\phi_{t}:=\phi e^{-i \int_{0}^{t}|u(s)|^{2} d s}$ is an inner function. Note that $\phi_{t}$ is well defined on $\mathbb{R}$ and its absolute value is 1 on $\mathbb{R}$. Consider the function defined by $h(x)=\phi_{t}(x) /(x+i)$ for $x \in \mathbb{R}$. Since $h \in L_{+}^{2}$, we can write using the Poisson integral that

$$
h(z)=\frac{1}{\pi} \int_{-\infty}^{\infty} \operatorname{Im} z \frac{h(x)}{|z-x|^{2}} d x
$$

for all $z \in \mathbb{C}_{+}$. Then,

$$
z h(z)=\frac{1}{\pi} \int_{-\infty}^{\infty} \operatorname{Im} z \frac{x h(x)}{|z-x|^{2}} d x+\frac{1}{\pi} \int_{-\infty}^{\infty} \operatorname{Im} z \frac{(z-x) h(x)}{|z-x|^{2}} d x .
$$

Note that the last integral is equal to

$$
\int_{-\infty}^{\infty} \operatorname{Im} z \frac{h(x)}{\bar{z}-x} d x
$$

By the residue theorem and using the fact that the function $h /(\bar{z}-x)$ is holomorphic on $\mathbb{C}_{+}$, we have that this integral is zero and thus

$$
z h(z)=\frac{1}{\pi} \int_{-\infty}^{\infty} \operatorname{Im} z \frac{x h(x)}{|z-x|^{2}} d x .
$$

Therefore, we can use the Poisson integral to extend $\phi_{t}$ to $\mathbb{C}_{+}$as a holomorphic function.

$$
\phi_{t}(z)=(z+i) h(z)=\frac{1}{\pi} \int_{-\infty}^{\infty} \operatorname{Im} z \frac{(x+i) h(x)}{|z-x|^{2}} d x=\frac{1}{\pi} \int_{-\infty}^{\infty} \operatorname{Im} z \frac{\phi_{t}(x)}{|z-x|^{2}} d x .
$$

Moreover,

$$
\left|\phi_{t}(z)\right| \leq \frac{1}{\pi} \int_{-\infty}^{\infty} \operatorname{Im} z \frac{1}{|z-x|^{2}} d x=1,
$$

for all $z \in \mathbb{C}_{+}$. Hence $\phi_{t}$ is an inner function.

Since $\tau_{c t}(\phi)$ and $\phi e^{-i \int_{0}^{t}|u(s)|^{2} d s}$ are inner functions and

$$
\phi e^{-i \int_{0}^{t}|u(s)|^{2} d s} L_{+}^{2}=\tau_{c t}(\phi) L_{+}^{2},
$$

Proposition 4.4 yields the existence of a real-valued function $\gamma$ such that $\gamma(0)=0$ and

$$
\phi e^{-i \int_{0}^{t}|u(s)|^{2} d s}=\tau_{c t}(\phi) e^{i \gamma(t)} .
$$

Taking the derivative with respect to $t$, we obtain that $\phi$ satisfies the equation

$$
c D \phi(x)=|u(t, x+c t)|^{2} \phi(x)+\dot{\gamma}(t) \phi(x) .
$$

for all $t \in \mathbb{R}$. Since $u$ is a traveling wave, we have $|u(t, x+c t)|=\left|e^{-i \omega t} u_{0}(x)\right|=\left|u_{0}(x)\right|$. Then we deduce that $\dot{\gamma}(t)=k$ and hence $\gamma(t)=k t$, for some $k \in \mathbb{R}$. Therefore,

$$
c D \phi=\left(\left|u_{0}\right|^{2}+k\right) \phi \text {. }
$$


We prove in the following that $k=0$. First, note that $\frac{k}{c} \geq 0$. The function $\phi u_{0} \in \operatorname{Ker} H_{u_{0}}$ and by Lemma 4.2, we have $\left|u_{0}\right|^{2} \phi=\bar{u}_{0}\left(u_{0} \phi\right) \in L_{+}^{2}$. If $k / c$ is negative, setting $\chi:=\frac{1}{c}\left|u_{0}\right|^{2} \phi \in L_{+}^{2}$ and passing into Fourier space, we have

$$
\hat{\phi}(\xi)=\frac{1}{\xi-k / c} \hat{\chi}(\xi) \mathbf{1}_{[0, \infty)}(\xi)
$$

This implies that $\phi \in L_{+}^{2}$, contradicting $|\phi(x)|=1$ for all $x \in \mathbb{R}$.

Let us now prove that $k / c=0$. Let $h \in L_{+}^{2}$ be regular. Then $\phi h \in \operatorname{Ker} H_{u_{0}}$, and by (4-7) we have

$$
A_{u_{0}}(\phi h)=\left(D-\frac{1}{c}\left|u_{0}\right|^{2}\right)(\phi h)=\phi\left(D-\frac{1}{c}\left|u_{0}\right|^{2}\right)(h)+h D \phi=\phi\left(D+\frac{k}{c}\right) h .
$$

Denoting by $\mu_{\phi h}\left(A_{u_{0}}\right)$ the spectral measure corresponding to $\phi h$, we have

$$
\begin{aligned}
\int f d \mu_{\phi h} & =\left(\phi h, f\left(A_{u_{0}}\right) \phi h\right)=\left(\phi h, \phi f\left(D+\frac{k}{c}\right) h\right)=\left(h, f\left(D+\frac{k}{c}\right) h\right) \\
& =\frac{1}{2 \pi} \int_{0}^{\infty} f\left(\xi+\frac{k}{c}\right)|\hat{h}(\xi)|^{2} d \xi=\frac{1}{2 \pi} \int_{k / c}^{\infty} f(\eta)\left|\hat{h}\left(\eta-\frac{k}{c}\right)\right|^{2} d \eta
\end{aligned}
$$

Consequently, supp $\mu_{\phi h}\left(A_{u_{0}}\right) \subset[k / c,+\infty)$. By Proposition 3.9, we have $\mathscr{H}_{\mathrm{ac}}\left(A_{u_{0}}\right) \subset \operatorname{Ker} H_{u_{0}}$, and therefore

$$
\sigma_{\mathrm{ac}}\left(A_{u_{0}}\right)=\bigcup_{\psi \in \mathscr{H}_{\mathrm{ac}}\left(A_{u_{0}}\right)} \operatorname{supp} \mu_{\psi} \subset \overline{\bigcup_{\phi h \in \operatorname{Ker} H_{u_{0}}} \operatorname{supp} \mu_{\phi h}} \subset\left[\frac{k}{c}, \infty\right) .
$$

Since, by Corollary 3.4, $\sigma_{\mathrm{ac}}\left(A_{u_{0}}\right)=[0, \infty)$, this yields $k=0$.

Proposition 4.6. All traveling waves are rational functions.

Proof. We first prove that $\phi$ is a Blaschke product.

Since $\phi$ is an inner function in the sense of Beurling and Lax, it has the canonical decomposition

$$
\phi(z)=\lambda B(z) e^{i a z} \exp \left(i \int_{\mathbb{R}} \frac{1+t z}{t-z} d v(t)\right),
$$

where $z \in \mathbb{C}_{+}, \lambda$ is a complex number of absolute value $1, a \geq 0, B$ is a Blaschke product having exactly the same zeroes as $\phi$, and $v$ is a positive singular measure with respect to the Lebesgue measure.

Because $\phi$ satisfies (4-4) and $u_{0} \in L^{\infty}(\mathbb{R})$, we obtain that $\phi$ has bounded derivative on $\mathbb{R}$ and hence it is uniformly continuous on $\mathbb{R}$. Then, since $\phi$ satisfies the Poisson formula (4-6), it follows that

$$
\phi(x+i \varepsilon) \rightarrow \phi(x), \text { as } \varepsilon \rightarrow 0,
$$

uniformly for $x \in \mathbb{R}$. Since $\phi$ is uniformly continuous on $\mathbb{R}$ and since $|\phi(x)|=1$ for all $x \in \mathbb{R}$, we deduce that the zeroes of $\phi$, and hence those of the Blaschke product $B$ as well, lie outside a strip $\left\{z \in \mathbb{C} ; 0 \leq \operatorname{Im} z \leq \varepsilon_{0}\right\}$, for some $\varepsilon_{0}>0$. Therefore, we have

$$
\frac{\phi(x+i \varepsilon)}{B(x+i \varepsilon)} \rightarrow \frac{\phi(x)}{B(x)} \quad \text { as } \varepsilon \rightarrow 0
$$


uniformly for $x$ in compact subsets of $\mathbb{R}$. Taking the logarithm of the absolute value and noticing that $|\phi(x) / B(x)|=1$, we obtain

$$
\int_{\mathbb{R}} \frac{\varepsilon}{(x-t)^{2}+\varepsilon^{2}} d v(t) \rightarrow 0
$$

uniformly for $x$ in compact subsets in $\mathbb{R}$. In particular, for all $\delta>0$ there exists $0<\varepsilon_{1} \leq \varepsilon_{0}$ such that for all $0<\varepsilon \leq \varepsilon_{1}$ and for all $x \in[0,1]$, we have

$$
\frac{1}{2 \varepsilon} v([x-\varepsilon, x+\varepsilon]) \leq \int_{x-\varepsilon}^{x+\varepsilon} \frac{\varepsilon}{(x-t)^{2}+\varepsilon^{2}} d \nu(t) \leq \int_{\mathbb{R}} \frac{\varepsilon}{(x-t)^{2}+\varepsilon^{2}} d \nu(t) \leq \delta .
$$

Taking $\varepsilon=\frac{1}{2 N} \leq \varepsilon_{1}$ with $N \in \mathbb{N}^{*}$, we obtain

$$
v([0,1])=v\left(\bigcup_{k=0}^{N-1}\left[\frac{k}{N}, \frac{k+1}{N}\right]\right) \leq N \delta \frac{1}{N}=\delta .
$$

In conclusion, $v([0,1])=0$, and one can prove similarly that the measure $v$ of any compact interval in $\mathbb{R}$ is zero. Hence $v \equiv 0$.

Consequently, $\phi(x)=\lambda B(x) e^{i a x}$ for all $x \in \mathbb{R}$. On the other hand, because $\phi$ satisfies (4-4), we have $\phi(x)=\phi(0) e^{(i / c) \int_{0}^{x}\left|u_{0}\right|^{2}}$ and, in particular, $\lim _{x \rightarrow \infty} \phi(x)=\phi(0) e^{(i / c) \int_{0}^{\infty}\left|u_{0}\right|^{2}}$. Since $\lim _{x \rightarrow \infty} B(x)=$ 1 , we conclude that $a=0$. Substituting $\phi=\lambda B$ (4-4), we obtain

$$
\frac{c}{i} \frac{B^{\prime}}{B}=\left|u_{0}\right|^{2}
$$

Then

$$
\frac{1}{i} \int_{-\infty}^{\infty} \frac{B^{\prime}(x)}{B(x)} d x<\infty
$$

Computing this integral, we obtain that

$$
\frac{1}{i} \int_{-\infty}^{\infty} \frac{B^{\prime}(x)}{B(x)} d x=2 \sum_{j} \int_{-\infty}^{\infty} \frac{\operatorname{Im} \lambda_{j}}{\left|x-\lambda_{j}\right|^{2}} d x=2 \sum_{j} \pi
$$

and thus it is finite if and only if $B$ is a finite Blaschke product, $B(x)=\prod_{j=1}^{N} \varepsilon_{j} \frac{x-\lambda_{j}}{x-\bar{\lambda}_{j}}$.
We prove that the traveling wave $u$ is a rational function. We have

$$
\text { Ker } H_{u}=\phi L_{+}^{2}=B L_{+}^{2} \text {. }
$$

Set $Y:=\operatorname{span}_{\mathbb{C}}\left\{\frac{1}{x-\bar{\lambda}_{j}}\right\}_{j=1}^{N}$; we show that $B L_{+}^{2}=Y^{\perp}$. Indeed, $f \in Y^{\perp}$ if and only if

$$
f\left(\lambda_{j}\right)=\frac{1}{2 \pi} \int_{\mathbb{R}} e^{i \xi \lambda_{j}} \widehat{f}(\xi) d \xi=\frac{1}{2 \pi}\left(\hat{f}, e^{-i \bar{\lambda}_{j} \xi}\right)=\left(f, \frac{1}{x-\bar{\lambda}_{j}}\right)=0,
$$

if and only if there exists $h \in L_{+}^{2}$ such that $f=B h$. Hence Ker $H_{u}=Y^{\perp}$. This yields $\overline{\operatorname{Ran}}_{u}=Y$. By Remark 2.2 it follows that $u$ is a rational function. More precisely, $u \in \operatorname{Ran} H_{u}=Y$.

Proposition 4.7. If $u$ is a traveling wave, there exists $\lambda>0$ such that $H_{u}^{2} u=\lambda u$. 
Proof. According to Remark 2.2, since $u$ is a rational function, we have $u \in \operatorname{Ran} H_{u}$.

Secondly, $u$ satisfies the equation of the traveling waves (1-4), which is equivalent to $A_{u}(u)=-\frac{\omega}{c} u$. Therefore, $u$ is an eigenfunction of the operator $A_{u}$ for the eigenvalue $-\omega / c$. Applying the identity $(1-10)$,

$$
A_{u} H_{u}+H_{u} A_{u}+\frac{\omega}{c} H_{u}+\frac{1}{c} H_{u}^{3}=0,
$$

to $u$ and then to $H_{u} u$, one deduces that $A_{u} H_{u}^{2} u=-\frac{\omega}{c} H_{u}^{2} u$. Therefore, the conclusion of the proposition follows once we prove all the eigenfunctions of the operator $A_{u}$ belonging to Ran $H_{u}$, corresponding to the same eigenvalue, are linearly dependent.

Let $a$ be en eigenvalue of the operator $A_{u}$ and let $\psi_{1}, \psi_{2} \in \operatorname{Ker}\left(A_{u}-a\right) \cap \operatorname{Ran} H_{u}$. Since $u$ is a rational function, by the Kronecker type Theorem $2.1, \psi_{1}$ and $\psi_{2}$ are also nonconstant rational functions. Then, one can find $\alpha, \beta \in \mathbb{C},(\alpha, \beta) \neq(0,0)$, such that $\psi:=\alpha \psi_{1}+\beta \psi_{2}=O\left(\frac{1}{x^{2}}\right)$ as $x \rightarrow \infty$. Moreover, we have $\psi \in L^{1}(\mathbb{R}), x \psi \in L^{2}(\mathbb{R})$, and thus we can compute $A_{u}(x \psi)$.

Passing into Fourier space we have

$$
\widehat{\Pi(x f)}(\xi)=i\left(\partial_{\xi} \hat{f}\right) \mathbf{1}_{\xi \geq 0}=i \partial_{\xi}\left(\hat{f} \mathbf{1}_{\xi \geq 0}\right)-i \hat{f}(\xi) \delta_{\xi=0}=\widehat{x \Pi f}(\xi)-i \hat{f}(0) \delta_{\xi=0},
$$

for all $f \in L^{1}(\mathbb{R})$. Thus, we obtain $\Pi(x f)=x \Pi(f)+\frac{1}{2 \pi i} \hat{f}(0)$ for all $f \in L^{1}(\mathbb{R})$. We then have

$$
A_{u}(x \psi)=x A_{u}(\psi)+\frac{1}{i} \psi-\frac{1}{2 c \pi i} \int_{\mathbb{R}}|u|^{2} \psi d x
$$

and therefore, since $A_{u} \psi=a \psi$,

$$
A_{u}(x \psi)=a x \psi+\frac{1}{i} \psi-\frac{1}{2 c \pi i} \int_{\mathbb{R}}|u|^{2} \psi d x .
$$

Since $x \psi \in \operatorname{Ran} H_{u}$ and $A_{u}\left(\operatorname{Ran} H_{u}\right) \subset \operatorname{Ran} H_{u}$ by Lemma 3.7, we have $A_{u}(x \psi) \in \operatorname{Ran} H_{u} \subset L^{2}(\mathbb{R})$. The constant in (4-9) is zero because all the other terms are in $L^{2}(\mathbb{R})$. Then we have

$$
\left(A_{u}-a\right)(x \psi)=\frac{1}{i} \psi .
$$

Applying the self-adjoint operator $A_{u}-a$ to both sides of (4-10), we obtain $\left(A_{u}-a\right)^{2}(x \psi)=0$ and

$$
\left\|\left(A_{u}-a\right)(x \psi)\right\|_{L^{2}}^{2}=\left(\left(A_{u}-a\right)(x \psi),\left(A_{u}-a\right)(x \psi)\right)=\left(\left(A_{u}-a\right)^{2}(x \psi), x \psi\right)=0 .
$$

Thus, $\left(A_{u}-a\right)(x \psi)=0$. In conclusion, by (4-10), $\psi=0$ and therefore all the eigenfunctions belonging to Ran $H_{u}$, corresponding to the same eigenvalue $a$, are linearly dependent.

Proof of Theorem 1.2. Since $u \in \operatorname{Ran} H_{u}$, there exists a unique function $g \in \operatorname{Ran} H_{u}$ such that $u=H_{u}(g)$. By Proposition 4.7, it results that $H_{u}(u)=\lambda g$. Applying the identity (1-10),

$$
A_{u} H_{u}+H_{u} A_{u}+\frac{\omega}{c} H_{u}+\frac{1}{c} H_{u}^{3}=0,
$$

to $g$ and using $A_{u} u=-\frac{\omega}{c} u$, one obtains

$$
H_{u}\left(A_{u} g+\frac{\lambda}{c} g\right)=0
$$


Since $A_{u}\left(\operatorname{Ran} H_{u}\right) \subset \operatorname{Ran} H_{u}$, we have

$$
A_{u} g+\frac{\lambda}{c} g \in \operatorname{Ran} H_{u} \cap \operatorname{Ker} H_{u}
$$

Therefore, $A_{u} g+\frac{\lambda}{c} g=0$, which is equivalent to

$$
c D g-T_{|u|^{2}} g+\lambda g=0 .
$$

We next find a simpler version of this equation, in order to determine the function $g$ explicitly. Note that $\bar{u}(1-g) \in L_{+}^{2}$, since it is orthogonal to each complex conjugate of a holomorphic function $f \in L_{+}^{2}$ :

$$
(\bar{u}(1-g), \bar{f})=(f(1-g), u)=(f, u)-\left(f, H_{u}(g)\right)=0 .
$$

Thus,

$$
T_{|u|^{2}}(g)=\Pi\left(|u|^{2}\right)-\Pi\left(|u|^{2}(1-g)\right)=H_{u}(u)-|u|^{2}(1-g)=\lambda g-|u|^{2}(1-g) .
$$

Passing into Fourier space and using the fact that $|u|^{2}$ is a real-valued function, one can write

$$
|u|^{2}=\int_{0}^{\infty} e^{i x \xi} \widehat{|u|^{2}}(\xi) d \xi+\int_{0}^{\infty} e^{-i x \xi \overline{|u|^{2}}}(\xi) d \xi=\Pi\left(|u|^{2}\right)+\overline{\Pi\left(|u|^{2}\right)}
$$

Therefore $|u|^{2}=H_{u}(u)+\overline{H_{u}(u)}=\lambda(g+\bar{g})$. Consequently, $T_{|u|^{2}}(g)=\lambda\left(-\bar{g}+g^{2}+|g|^{2}\right)$ and $g$ solves the equation

$$
c D g-\lambda g^{2}+\lambda\left(g+\bar{g}-|g|^{2}\right)=0
$$

We prove that $g+\bar{g}-|g|^{2}=0$. First, note that $\bar{u}(1-g) \in L_{+}^{2}$, also yields $(1-g) f \in \operatorname{Ker} H_{u}$, for all $f \in L_{+}^{2}$. Secondly, let us prove that $g+\bar{g}-|g|^{2}$ is orthogonal to the complex conjugate of all $f \in L_{+}^{2}$ :

$$
\left(g+\bar{g}-|g|^{2}, \bar{f}\right)=(g, \bar{f})-(f(1-g), g)=-\left(f(1-g), \frac{1}{\lambda} H_{u}(u)\right)=-\frac{1}{\lambda}\left(u, H_{u}(f(1-g))\right)=0
$$

In addition, since $g+\bar{g}-|g|^{2}$ is a real-valued function, we have

$$
\left(g+\bar{g}-|g|^{2}, f\right)=\left(g+\bar{g}-|g|^{2}, \bar{f}\right)=0
$$

for all $f \in L_{+}^{2}$. Therefore, $g+\bar{g}-|g|^{2}$ is orthogonal to all the functions in $L^{2}(\mathbb{R})$ and thus $g+\bar{g}-|g|^{2}=0$. This is equivalent to $|1-g|=1$ on $\mathbb{R}$. Moreover, (4-11) gives the precise formula for $g$ :

$$
g(z)=\frac{r}{z-p}
$$

where $r, p \in \mathbb{C}$ and $\operatorname{Im}(p)<0$. Thus $1-g(x)=\frac{x-\bar{p}}{x-p}$ for all $x \in \mathbb{R}$ and

$$
\operatorname{Ker} H_{\frac{1}{z-p}}=\frac{z-\bar{p}}{z-p} L_{+}^{2}=(1-g) L_{+}^{2} \subset \operatorname{Ker} H_{u} .
$$

Consequently, $u \in \operatorname{Ran} H_{u} \subset \operatorname{Ran} \frac{H_{\frac{1}{z-p}}}{z-p}$. 


\section{Orbital stability of traveling waves}

In order to prove the orbital stability of traveling waves, we first use the fact that they are minimizers of the Gagliardo-Nirenberg inequality. We begin this section by proving this inequality:

Proof of Proposition 1.5, the Gagliardo-Nirenberg inequality. The proof is similar to that of the GagliardoNirenberg inequality for the circle, in [Gérard and Grellier 2010b]. The idea is to write all the norms in the Fourier space, using Plancherel's identity.

$$
E=\|u\|_{L^{4}}^{4}=\left\|u^{2}\right\|_{L^{2}}^{2}=\frac{1}{2 \pi}\left\|\widehat{u^{2}}\right\|_{L^{2}}^{2}=\frac{1}{2 \pi} \int_{\mathbb{R}}\left|\widehat{u^{2}}(\xi)\right|^{2} d \xi
$$

Using the fact that $u \in L_{+}^{2}$ and Cauchy-Schwarz inequality, we have

$$
\begin{aligned}
\left|\widehat{u^{2}}(\xi)\right|^{2} & =\frac{1}{4 \pi^{2}}\left|\int_{0}^{\xi} \widehat{u}(\eta) \widehat{u}(\xi-\eta) d \eta\right|^{2} \leq \frac{1}{4 \pi^{2}} \xi \int_{0}^{\xi}|\widehat{u}(\eta)|^{2}|\widehat{u}(\xi-\eta)|^{2} d \eta \\
& \leq \frac{1}{4 \pi^{2}}\left(\int_{0}^{\xi} \eta|\widehat{u}(\eta)|^{2}|\widehat{u}(\xi-\eta)|^{2} d \eta+\int_{0}^{\xi}(\xi-\eta)|\widehat{u}(\eta)|^{2}|\widehat{u}(\xi-\eta)|^{2} d \eta\right) .
\end{aligned}
$$

By the change of variables $\xi-\eta \mapsto \eta$ in the second integral, we have

$$
\left|\widehat{u^{2}}(\xi)\right|^{2} \leq \frac{1}{2 \pi^{2}} \int_{0}^{\xi} \eta|\widehat{u}(\eta)|^{2}|\widehat{u}(\xi-\eta)|^{2} d \eta .
$$

By Fubini's theorem and change of variables $\zeta=\xi-\eta$ it results that

$$
E \leq \frac{1}{4 \pi^{3}} \int_{\mathbb{R}} \int_{0}^{\xi} \eta|\widehat{u}(\eta)|^{2}|\widehat{u}(\xi-\eta)|^{2} d \eta d \xi=\left.\frac{1}{4 \pi^{3}} \int_{0}^{+\infty} \eta \widehat{u}(\eta)\right|^{2} d \eta \int_{0}^{+\infty}|\widehat{u}(\zeta)|^{2} d \zeta=\frac{1}{\pi} M Q .
$$

Equality holds if and only if we have equality in Cauchy-Schwarz inequality, i.e.

$$
\widehat{u}(\xi) \widehat{u}(\eta)=\widehat{u}(\xi+\eta) \widehat{u}(0),
$$

for all $\xi, \eta \geq 0$. This is true if and only if

$$
\widehat{u}(\xi)=e^{-i p \xi} \widehat{u}(0) \text { for all } \xi \geq 0 .
$$

Since $u \in H_{+}^{1 / 2}$, this yields $\operatorname{Im}(p)<0$ and $u(x)=C /(x-p)$, for some constant $C$.

The second argument we use in proving the stability of traveling waves is a profile decomposition theorem. It states that bounded sequences in $H_{+}^{1 / 2}$ can be written as superposition of translations of fixed profiles and of a remainder term. The remainder is small in all the $L^{p}$-norms, $2<p<\infty$. Moreover, the superposition is almost orthogonal in the $H_{+}^{1 / 2}$-norm.

Proposition 5.1 (Profile decomposition theorem for bounded sequences in $H_{+}^{1 / 2}$ ). Let $\left\{v^{n}\right\}_{n \in \mathbb{N}}$ be $a$ bounded sequence in $H_{+}^{1 / 2}$. There exist a subsequence of $\left\{v^{n}\right\}_{n \in \mathbb{N}}$, still denoted by $\left\{v^{n}\right\}_{n \in \mathbb{N}}$, a sequence of fixed profiles in $H_{+}^{1 / 2},\left\{V^{(j)}\right\}_{j \in \mathbb{N}}$, and a family of real sequences $\left\{x^{(j)}\right\}_{j \in \mathbb{N}}$ such that for all $\ell \in \mathbb{N}^{*}$ we have

$$
v^{n}=\sum_{j=1}^{\ell} V^{(j)}\left(x-x_{n}^{(j)}\right)+r_{n}^{(\ell)},
$$


where

$$
\lim _{\ell \rightarrow \infty} \limsup _{n \rightarrow \infty}\left\|r_{n}^{(\ell)}\right\|_{L^{p}(\mathbb{R})}=0
$$

for all $p \in(2, \infty)$, and

$$
\begin{aligned}
\left\|v^{n}\right\|_{L^{2}}^{2} & =\sum_{j=1}^{\ell}\left\|V^{(j)}\right\|_{L^{2}}^{2}+\left\|r_{n}^{(\ell)}\right\|_{L^{2}}^{2}+o(1) \quad \text { as } n \rightarrow \infty \\
\left\|v^{n}\right\|_{\dot{H}_{+}^{1 / 2}}^{2} & =\sum_{j=1}^{\ell}\left\|V^{(j)}\right\|_{\dot{H}_{+}^{1 / 2}}^{2}+\left\|r_{n}^{(\ell)}\right\|_{\dot{H}_{+}^{1 / 2}}^{2}+o(1) \text { as } n \rightarrow \infty \\
\lim _{n \rightarrow \infty}\left\|v^{n}\right\|_{L^{4}}^{4} & =\sum_{j=1}^{\infty}\left\|V^{(j)}\right\|_{L^{4}}^{4} .
\end{aligned}
$$

The proof of this proposition follows exactly the same lines as that of the profile decomposition theorem for bounded sequences in $H^{1}(\mathbb{R})$; see [Hmidi and Keraani 2006, Proposition 2.1]. However, note that in our case, the profiles $V^{(j)}$ belong to the space $H_{+}^{1 / 2}$ (not only to the space $H^{1 / 2}(\mathbb{R})$ ), as they are weak limits of translations of the sequence $\left\{v^{n}\right\}_{n \in \mathbb{N}}$.

Proof of Theorem 1.3. According to Proposition 1.5, $C(a, r)$ is the set of minimizers of the problem

$$
\inf \left\{M(u) ; u \in H_{+}^{1 / 2}, Q(u)=q(a, r), E(u)=e(a, r)\right\},
$$

where

$$
q(a, r)=\frac{a^{2} \pi}{r}, \quad e(a, r)=\frac{a^{4} \pi}{2 r^{3}} .
$$

We denote the infimum by $m(a, r)$. Since

$$
\inf _{\phi \in C(a, r)}\left\|u_{0}^{n}-\phi\right\|_{H_{+}^{1 / 2}} \rightarrow 0
$$

by the Sobolev embedding theorem, we deduce

$$
Q\left(u_{0}^{n}\right) \rightarrow q(a, r), \quad E\left(u_{0}^{n}\right) \rightarrow e(a, r), \quad M\left(u_{0}^{n}\right) \rightarrow m(a, r) .
$$

Let $\left\{t_{n}\right\}_{n \in \mathbb{N}}$ be an arbitrary sequence of real numbers. The conservation laws yield

$$
Q\left(u^{n}\left(t_{n}\right)\right) \rightarrow q(a, r), \quad E\left(u^{n}\left(t_{n}\right)\right) \rightarrow e(a, r), \quad M\left(u^{n}\left(t_{n}\right)\right) \rightarrow m(a, r) .
$$

We can choose two sequences of positive numbers $\left\{a_{n}\right\}$ and $\left\{\lambda_{n}\right\}$ such that $v^{n}(x):=a_{n} u^{n}\left(t_{n}, \lambda_{n} x\right)$ satisfies $\left\|v^{n}\right\|_{L^{2}(\mathbb{R})}=1,\left\|v^{n}\right\|_{L^{4}(\mathbb{R})}=1$. Notice that

$$
a_{n} \rightarrow a_{\infty}, \quad \lambda_{n} \rightarrow \lambda_{\infty}
$$

where $a_{\infty}>0, \lambda_{\infty}>0$, and

$$
\frac{\lambda_{\infty}}{a_{\infty}^{4}}=e(a, r), \quad \frac{\lambda_{\infty}}{a_{\infty}^{2}}=q(a, r) .
$$


Then

$$
\left\|v^{n}\right\|_{\dot{H}_{+}^{1 / 2}}^{1 / 2}=\frac{\left\|v^{n}\right\|_{L^{2}}^{1 / 2}\left\|v^{n}\right\|_{\dot{H}_{+}^{1 / 2}}^{1 / 2}}{\left\|v^{n}\right\|_{L^{4}}}=\frac{\left\|u^{n}\left(t_{n}\right)\right\|_{L^{2}}^{1 / 2}\left\|u^{n}\left(t_{n}\right)\right\|_{\dot{H}_{+}^{1 / 2}}^{1 / 2}}{\left\|u^{n}\left(t_{n}\right)\right\|_{L^{4}}} \text { for all } n \in \mathbb{N} .
$$

In particular, as a consequence of the Gagliardo-Nirenberg inequality,

$$
\lim _{n \rightarrow \infty}\left\|v^{n}\right\|_{\dot{H}_{+}^{1 / 2}}=\sqrt{\pi}
$$

Thus the sequence $\left\{v^{n}\right\}_{n \in \mathbb{N}}$ is bounded in $H_{+}^{1 / 2}$. From the profile decomposition theorem (Proposition 5.1), we obtain that there exist real sequences $\left\{x^{(j)}\right\}_{j \in \mathbb{N}}$ depending on the sequence $\left\{t_{n}\right\}_{n \in \mathbb{N}}$ in the definition of $\left\{v^{n}\right\}_{n \in \mathbb{N}}$, such that for all $\ell \in \mathbb{N}^{*}$ we have

$$
v^{n}=\sum_{j=1}^{\ell} V^{(j)}\left(x-x_{n}^{(j)}\right)+r_{n}^{(\ell)}
$$

where

$$
\lim _{\ell \rightarrow \infty} \limsup _{n \rightarrow \infty}\left\|r_{n}^{(\ell)}\right\|_{L^{p}(\mathbb{R})}=0
$$

for all $p \in(2, \infty)$, and

$$
\begin{aligned}
\left\|v^{n}\right\|_{L^{2}}^{2} & =\sum_{j=1}^{\ell}\left\|V^{(j)}\right\|_{L^{2}}^{2}+\left\|r_{n}^{(\ell)}\right\|_{L^{2}}^{2}+o(1) \quad \text { as } n \rightarrow \infty \\
\left\|v^{n}\right\|_{\dot{H}_{+}^{1 / 2}}^{2} & =\sum_{j=1}^{\ell}\left\|V^{(j)}\right\|_{\dot{H}_{+}^{1 / 2}}^{2}+\left\|r_{n}^{(\ell)}\right\|_{\dot{H}_{+}^{1 / 2}}^{2}+o(1) \quad \text { as } n \rightarrow \infty \\
\lim _{n \rightarrow \infty}\left\|v^{n}\right\|_{L^{4}}^{4} & =\sum_{j=1}^{\infty}\left\|V^{(j)}\right\|_{L^{4}}^{4} .
\end{aligned}
$$

Consequently,

$$
1 \geq \sum_{j=1}^{\infty}\left\|V^{(j)}\right\|_{L^{2}}^{2}, \quad \pi \geq \sum_{j=1}^{\infty}\left\|V^{(j)}\right\|_{\dot{H}_{+}^{1 / 2}}^{2}, \quad 1=\sum_{j=1}^{\infty}\left\|V^{(j)}\right\|_{L^{4}}^{4} .
$$

Therefore, by the Gagliardo-Nirenberg inequality (1-12), we have

$$
\pi \geq\left(\sum_{j=1}^{\infty}\left\|V^{(j)}\right\|_{L^{2}}^{2}\right)\left(\sum_{j=1}^{\infty}\left\|V^{(j)}\right\|_{\dot{H}_{+}^{1 / 2}}^{2}\right) \geq \sum_{j=1}^{\infty}\left\|V^{(j)}\right\|_{L^{2}}^{2}\left\|V^{(j)}\right\|_{\dot{H}_{+}^{1 / 2}}^{2} \geq \pi \sum_{j=1}^{\infty}\left\|V^{(j)}\right\|_{L^{4}}^{4}=\pi .
$$

Thus, there exist only one profile $V:=V^{(1)}$ and a sequence $x=x^{(1)}$ such that

$$
\begin{array}{rlrl}
v^{n} & =V\left(x-x_{n}\right)+r_{n}, \\
\left\|v^{n}\right\|_{L^{2}}^{2} & =\|V\|_{L^{2}}^{2}+\left\|r_{n}\right\|_{L^{2}}^{2}+o(1) \quad \text { as } n \rightarrow \infty, \\
\left\|v^{n}\right\|_{\dot{H}_{+}^{1 / 2}}^{2} & =\|V\|_{\dot{H}_{+}^{1 / 2}}^{2}+\left\|r_{n}\right\|_{\dot{H}_{+}^{1 / 2}}^{2}+o(1) & \text { as } n \rightarrow \infty .
\end{array}
$$


According to (5-1), $V$ satisfies $1 \geq\|V\|_{L^{2}}^{2}, \pi \geq\|V\|_{\dot{H}_{+}^{1 / 2}}^{2}$, and $\|V\|_{L^{4}}^{4}=1$. In conclusion,

$$
\pi=\pi\|V\|_{L^{4}}^{4} \leq\|V\|_{L^{2}}^{2}\|V\|_{\dot{H}_{+}^{1 / 2}}^{2} \leq \pi .
$$

Hence, $V$ is a minimizer in the Gagliardo-Nirenberg inequality. Moreover,

$$
\|V\|_{L^{2}}^{2}=1=\left\|v^{n}\right\|_{L^{2}}, \quad\|V\|_{\dot{H}_{+}^{1 / 2}}^{2}=\pi=\lim _{n \rightarrow \infty}\left\|v^{n}\right\|_{\dot{H}_{+}^{1 / 2}}^{2} .
$$

By (5-2) and (5-3), we have $r_{n} \rightarrow 0$ in $H_{+}^{1 / 2}$ as $n \rightarrow \infty$. Consequently, $v^{n}\left(\cdot+x_{n}\right) \rightarrow V$ in $H_{+}^{1 / 2}$, or equivalently,

$$
\lim _{n \rightarrow \infty}\left\|a_{n} u^{n}\left(t_{n}, \lambda_{n} x\right)-V\left(x-x_{n}\right)\right\|_{H_{+}^{1 / 2}}=0
$$

We then have

$$
\lim _{n \rightarrow \infty}\left\|u^{n}\left(t_{n}, x\right)-\frac{1}{a_{\infty}} V\left(\frac{x-x_{n} \lambda_{\infty}}{\lambda_{\infty}}\right)\right\|_{H_{+}^{1 / 2}}=0 .
$$

Notice that, since $V$ is a minimizer in the Gagliardo-Nirenberg inequality, we have

$$
\tilde{\phi}(x):=\frac{1}{a_{\infty}} V\left(\frac{x}{\lambda_{\infty}}\right)=\frac{\alpha}{x-p} \in C(a, r) .
$$

Then, since $x_{n} \lambda_{\infty} \in \mathbb{R}$, we have $\phi(x)=\tilde{\phi}\left(x-x_{n} \lambda_{\infty}\right)=\frac{\alpha}{x-\tilde{p}} \in C(a, r)$. Thus,

$$
\inf _{\phi \in C(a, r)}\left\|u^{n}\left(t_{n}, x\right)-\phi(x)\right\|_{H_{+}^{1 / 2}} \rightarrow 0, \text { as } n \rightarrow \infty .
$$

The conclusion follows by approximating the supremum in the statement by the sequence in (5-4) with an appropriate $\left\{t_{n}\right\}_{n \in \mathbb{N}}$.

\section{Acknowledgments}

The author is grateful to her Ph.D. advisor, Prof. Patrick Gérard, for introducing her to this subject and for constantly supporting her during the preparation of this paper. She would also like to thank the referee for his helpful comments.

\section{References}

[Burq et al. 2005] N. Burq, P. Gérard, and N. Tzvetkov, "Bilinear eigenfunction estimates and the nonlinear Schrödinger equation on surfaces”, Invent. Math. 159:1 (2005), 187-223. MR 2005m:35275 Zbl 1092.35099

[Cazenave and Lions 1982] T. Cazenave and P.-L. Lions, "Orbital stability of standing waves for some nonlinear Schrödinger equations", Comm. Math. Phys. 85:4 (1982), 549-561. MR 84i:81015 Zbl 0513.35007

[Eckhaus and Schuur 1983] W. Eckhaus and P. Schuur, "The emergence of solitons of the Korteweg-de Vries equation from arbitrary initial conditions", Math. Methods Appl. Sci. 5:1 (1983), 97-116. MR 85a:35079 Zbl 0518.35074

[Gérard 1998] P. Gérard, "Description du défaut de compacité de l'injection de Sobolev", ESAIM Control Optim. Calc. Var. 3 (1998), 213-233. MR 99h:46051 Zbl 0907.46027

[Gérard and Grellier 2010a] P. Gérard and S. Grellier, “The cubic Szegó equation”, Ann. Sci. Éc. Norm. Supér. (4) 43:5 (2010), 761-810. MR 2721876 Zbl pre05939690

[Gérard and Grellier 2010b] P. Gérard and S. Grellier, "L'équation de Szegö cubique", in Séminaire X Équations aux dérivées partielles (Palaiseau, 2008), École Polytechnique, 2010. Zbl 1213.35397 
[Hmidi and Keraani 2006] T. Hmidi and S. Keraani, "Remarks on the blowup for the $L^{2}$-critical nonlinear Schrödinger equations", SIAM J. Math. Anal. 38:4 (2006), 1035-1047. MR 2008e:35184 Zbl 1122.35135

[Holmer and Zworski 2008] J. Holmer and M. Zworski, "Soliton interaction with slowly varying potentials", Int. Math. Res. Not. 2008:10 (2008), Art. ID rnn026. MR 2009i:37183 Zbl 1147.35084

[Hörmander 1990] L. Hörmander, The analysis of linear partial differential operators, I: Distribution theory and Fourier analysis, 2nd ed., Grundlehren der Math. Wiss. 256, Springer, Berlin, 1990. MR 91m:35001a Zbl 0712.35001

[Lax 1959] P. D. Lax, “Translation invariant spaces”, Acta Math. 101 (1959), 163-178. MR 21 \#4359 Zbl 0085.09102

[Nikolski 2002] N. K. Nikolski, Operators, functions, and systems: an easy reading, I: Hardy, Hankel, and Toeplitz, Mathematical Surveys and Monographs 92, American Mathematical Society, Providence, RI, 2002. MR 2003i:47001a Zbl 1007.47001

[Peller 2003] V. V. Peller, Hankel operators and their applications, Springer Monographs in Mathematics, Springer, New York, 2003. MR 2004e:47040 Zbl 1030.47002

[Perelman 2009] G. Perelman, "A remark on soliton-potential interactions for nonlinear Schrödinger equations", Math. Res. Lett. 16:3 (2009), 477-486. MR 2010k:35468 Zbl 1172.81012

[Reed and Simon 1979] M. Reed and B. Simon, Methods of modern mathematical physics, III: Scattering theory, Academic Press, New York, 1979. MR 58 \#12429a Zbl 0405.47007

[Rudin 1974] W. Rudin, Real and complex analysis, 2nd ed., McGraw-Hill, New York, 1974. MR 49 \#8783 Zbl 0278.26001

[Weinstein 1982] M. I. Weinstein, "Nonlinear Schrödinger equations and sharp interpolation estimates", Comm. Math. Phys. 87:4 (1982), 567-576. MR 84d:35140

Received 19 Jan 2010. Revised 28 Apr 2010. Accepted 29 May 2010.

OANA POCOVNICU: Dana.Pocovnicu@math.u-psud.fr

Laboratoire de Mathématiques d'Orsay, Université Paris-Sud (XI), Campus d'Orsay, bât. 430, 91405, Orsay, France 


\section{Analysis \& PDE}

pjm.math.berkeley.edu/apde

EDITORS

EDITOR-IN-CHIEF

Maciej Zworski

University of California

Berkeley, USA

BOARD OF EDITORS

\begin{tabular}{|c|c|c|c|}
\hline Michael Aizenman & $\begin{array}{l}\text { Princeton University, USA } \\
\text { aizenman@math.princeton.edu }\end{array}$ & Nicolas Burq & $\begin{array}{l}\text { Université Paris-Sud 11, France } \\
\text { nicolas.burq@math.u-psud.fr }\end{array}$ \\
\hline Luis A. Caffarelli & $\begin{array}{l}\text { University of Texas, USA } \\
\text { caffarel@math.utexas.edu }\end{array}$ & un-Yung Alice Chang & $\begin{array}{l}\text { Princeton University, USA } \\
\text { chang@math.princeton.edu }\end{array}$ \\
\hline Michael Christ & $\begin{array}{l}\text { University of California, Berkeley, USA } \\
\text { mchrist@math.berkeley.edu }\end{array}$ & Charles Fefferman & $\begin{array}{l}\text { Princeton University, USA } \\
\text { cf@math.princeton.edu }\end{array}$ \\
\hline Ursula Hamenstaedt & $\begin{array}{l}\text { Universität Bonn, Germany } \\
\text { ursula@math.uni-bonn.de }\end{array}$ & Nigel Higson & $\begin{array}{l}\text { Pennsylvania State Univesity, USA } \\
\text { higson@ math.psu.edu }\end{array}$ \\
\hline Vaughan Jones & $\begin{array}{l}\text { University of California, Berkeley, USA } \\
\text { vfr@math.berkeley.edu }\end{array}$ & Herbert Koch & $\begin{array}{l}\text { Universität Bonn, Germany } \\
\text { koch@math.uni-bonn.de }\end{array}$ \\
\hline Izabella Laba & $\begin{array}{l}\text { University of British Columbia, Canada } \\
\text { ilaba@math.ubc.ca }\end{array}$ & Gilles Lebeau & $\begin{array}{l}\text { Université de Nice Sophia Antipolis, France } \\
\text { lebeau@unice.fr }\end{array}$ \\
\hline László Lempert & $\begin{array}{l}\text { Purdue University, USA } \\
\text { lempert@math.purdue.edu }\end{array}$ & Richard B. Melrose & $\begin{array}{l}\text { Massachussets Institute of Technology, USA } \\
\text { rbm@math.mit.edu }\end{array}$ \\
\hline Frank Merle & $\begin{array}{l}\text { Université de Cergy-Pontoise, France } \\
\text { Frank.Merle@u-cergy.fr }\end{array}$ & William Minicozzi II & $\begin{array}{l}\text { Johns Hopkins University, USA } \\
\text { minicozz@ math.jhu.edu }\end{array}$ \\
\hline Werner Müller & $\begin{array}{l}\text { Universität Bonn, Germany } \\
\text { mueller@math.uni-bonn.de }\end{array}$ & Yuval Peres & $\begin{array}{l}\text { University of California, Berkeley, USA } \\
\text { peres@stat.berkeley.edu }\end{array}$ \\
\hline Gilles Pisier & $\begin{array}{l}\text { Texas A\&M University, and Paris } 6 \\
\text { pisier@math.tamu.edu }\end{array}$ & Tristan Rivière & $\begin{array}{l}\text { ETH, Switzerland } \\
\text { riviere@math.ethz.ch }\end{array}$ \\
\hline Igor Rodnianski & $\begin{array}{l}\text { Princeton University, USA } \\
\text { irod@math.princeton.edu }\end{array}$ & Wilhelm Schlag & $\begin{array}{l}\text { University of Chicago, USA } \\
\text { schlag@math.uchicago.edu }\end{array}$ \\
\hline Sylvia Serfaty & $\begin{array}{l}\text { New York University, USA } \\
\text { serfaty@ cims.nyu.edu }\end{array}$ & Yum-Tong Siu & $\begin{array}{l}\text { Harvard University, USA } \\
\text { siu@math.harvard.edu }\end{array}$ \\
\hline Terence Tao & $\begin{array}{l}\text { University of California, Los Angeles, USA } \\
\text { tao@math.ucla.edu }\end{array}$ & A Michael E. Taylor & $\begin{array}{l}\text { Univ. of North Carolina, Chapel Hill, USA } \\
\text { met@math.unc.edu }\end{array}$ \\
\hline Gunther Uhlmann & $\begin{array}{l}\text { University of Washington, USA } \\
\text { gunther@math.washington.edu }\end{array}$ & András Vasy & $\begin{array}{l}\text { Stanford University, USA } \\
\text { andras@math.stanford.edu }\end{array}$ \\
\hline Virgil Voicul & $\begin{array}{l}\text { University of California, Berkeley, USA } \\
\text { dvv@math.berkeley.edu }\end{array}$ & Steven Zelditch & $\begin{array}{l}\text { Northwestern University, USA } \\
\text { zelditch@math.northwestern.edu }\end{array}$ \\
\hline
\end{tabular}

PRODUCTION

contact@msp.org

Silvio Levy, Scientific Editor

Sheila Newbery, Senior Production Editor

See inside back cover or pjm.math.berkeley.edu/apde for submission instructions.

The subscription price for 2011 is US \$120/year for the electronic version, and \$180/year for print and electronic. Subscriptions, requests for back issues from the last three years and changes of subscribers address should be sent to Mathematical Sciences Publishers, Department of Mathematics, University of California, Berkeley, CA 94720-3840, USA.

Analysis \& PDE, at Mathematical Sciences Publishers, Department of Mathematics, University of California, Berkeley, CA 94720-3840 is published continuously online. Periodical rate postage paid at Berkeley, CA 94704, and additional mailing offices.

APDE peer review and production are managed by EditFLOW ${ }^{\mathrm{TM}}$ from Mathematical Sciences Publishers.

PUBLISHED BY

mathematical sciences publishers

http://msp.org/

A NON-PROFIT CORPORATION

Typeset in IATEX

Copyright $(2011$ by Mathematical Sciences Publishers 


\section{ANALYSIS \& PDE}

\section{Volume $4 \quad$ No. $3 \quad 2011$}

Regularity of weak solutions of a complex Monge-Ampère equation

GÁBOR SZÉKELYHIDI and VALENTINO TOSATTI

Traveling waves for the cubic Szegó equation on the real line

OANA POCOVNICU

Scattering threshold for the focusing nonlinear Klein-Gordon equation

405

Slim IBRAHIM, NADER MASMOUdi and KenJi NAKANISHI

Rayleigh-type surface quasimodes in general linear elasticity

463

SÖNKE HANSEN 